\title{
ALGEBRAIC TORI AS DEGENERATIONS OF ABELIAN VARIETIES
}

\author{
KAI-WEN LAN AND JUNECUE SUH
}

\begin{abstract}
We first show that every algebraic torus over any field, not necessarily split, can be realized as the special fiber of a semi-abelian scheme whose generic fiber is an absolutely simple abelian variety. Then we investigate which algebraic tori can be thus obtained, when we require the generic fiber of the semi-abelian scheme to carry non-trivial endomorphism structures.
\end{abstract}

\section{Contents}

1 Introduction

2 One-dimensional case

3 Descent data for tori

4 Theory of degeneration

5 Conditions for degenerations

6 Proof of main theorem

7 Non-trivial endomorphisms

\$1. Introduction. We start with a well-known example, the Dwork family of elliptic curves

$$
X^{3}+Y^{3}+Z^{3}-3 t X Y Z=0
$$

over the projective line $\mathbb{P}_{\mathbb{Q}}^{1}$ over $\mathbb{Q}$, where $t$ is the affine coordinate, with the identity section given by the point at infinity $[X: Y: Z]=[1:-1: 0]$. It also doubles as the modular curve of level 3.

It has bad, semistable reduction at $t=\infty$ and the third roots of unity. The corresponding Néron model has the split torus as the identity component of the fiber at $t=\infty$ (because the equation becomes $X Y Z=0$ ), but at $t=1$ we get a non-split torus $T$ that sits in the short exact sequence

$$
1 \longrightarrow T \longrightarrow \operatorname{Res}_{\mathbb{Q}(\sqrt{-3}) / \mathbb{Q}} \mathbb{G}_{\mathrm{m}} \stackrel{\text { Norm }}{\longrightarrow} \mathbb{G}_{\mathrm{m}} \longrightarrow 1,
$$

where Norm denotes the norm homomorphism of the quadratic extension $\mathbb{Q}(\sqrt{-3})$ over $\mathbb{Q}$. This leads to the following natural question.

Received 11 January 2017, published online 20 March 2018.

MSC (2010): 11G10, 14D06 (primary), 11G15, $11 \mathrm{G} 18$ (secondary).

The first author's research is partially supported by the NSF Grant DMS-1352216 and by an Alfred P. Sloan Research Fellowship. Any opinions, findings, and conclusions or recommendations expressed in this material are those of the authors and do not necessarily reflect the views of these organizations. 
Question 1.1. To which algebraic tori can abelian varieties degenerate?

However, this question turns out to be a bit naive, in the sense that it allows an answer that is somewhat shallow. Indeed, as we shall explain in the main text with more details, the question can be rather easily reduced to the case of the split one-dimensional torus $\mathbb{G}_{\mathrm{m}}$ by finite étale descent, where an affirmative answer is provided by the classical theory of Tate curves. The generic abelian variety obtained this way will be geometrically the $n$-fold product of an elliptic curve without CM (complex multiplication), where $n$ is the dimension of the torus.

A better and deeper question is the following.

Question 1.2. To which algebraic tori can absolutely simple abelian varieties degenerate?

Here, as usual, an abelian variety is called absolutely simple if its base change to an algebraic closure of the base field is not isogenous to a non-trivial product of abelian varieties of smaller dimensions.

For the distinction between Questions 1.1 and 1.2, an apt analogy can be made with complex moduli of abelian varieties. To a simple algebra $B$ with involution of types I-IV and a fixed complex representation of $B$, one attaches the moduli of polarized abelian varieties with endomorphisms of the given type. One expects, and can prove in most cases, that a very general member in the moduli has an endomorphism algebra no bigger than $B$; the rough idea is that those abelian varieties with more endomorphisms than prescribed should form a "thin" or nowhere dense subset. However, a careful proof is necessary, as there are exceptional cases where a very general member is not even simple (see [20, §4, Theorem 5 and Propositions 14, 15, 17, 18, and 19] or [2, Theorem 9.9.1]).

Our main theorem answers Question 1.2, in the affirmative, for all algebraic tori over all base fields.

THEOREM 1.3. Let $T$ be any algebraic torus over any base field $k$. Then there exists a semi-abelian scheme $G$ over a noetherian normal base scheme $S$ with a $k$-valued closed point $s \in S(k)$ generalizing to a generic point $\eta$ such that the following hold.

(1) The special fiber $G_{s}$ is isomorphic to $T$ over $k$.

(2) The generic fiber $G_{\eta}$ is an absolutely simple abelian variety.

More precisely, we may take $S$ to be of one of the following three kinds of Dedekind schemes:

(a) the spectrum of any complete discrete valuation ring, which can be freely prescribed (independent of $T$ ), with residue field $k$;

(b) a certain connected smooth curve over $k$ (depending on $T$ ) with a $k$-valued point $s$; and

(c) (when $k$ is a finite field) a certain "arithmetic curve" (depending on T), i.e. an open subset of the spectrum of some number ring, with a closed point $s$ corresponding to an unramified prime ideal with residue field $k$. 
In case $T$ has dimension one, we can exhibit the desired semi-abelian schemes $G$ over Dedekind $S$ for each non-split torus $T$ explicitly. Such tori are classified by the separable quadratic extensions of $k$, and we can write down suitable Weierstraß equations with special fibre $T$. (The generic absolute simpleness is automatic in dimension one.)

However, in dimensions $n \geqslant 2$ (already for $n=2$ ), we quickly lose explicit control on both sides. The isomorphism classes of $n$-dimensional tori over $k$ are in one-to-one correspondence with the isomorphism classes of continuous representations

$$
\rho: \operatorname{Gal}\left(k^{s} / k\right) \rightarrow \mathrm{GL}_{n}(\mathbb{Z})
$$

where $k^{s}$ denotes any separable closure of $k$, on the discrete module $\mathbb{Z}^{n}$ (corresponding to the Galois actions on the character groups of the tori), and it seems hopeless to classify them completely. On the other side, we do not have a good way of writing down families of general abelian varieties using explicit equations. In order to solve the problem, we use the theory of degeneration of polarized semi-abelian schemes (as in [18], [7, Chs II and III], and [14, Ch. 4]).

After the main theorem, it is natural to ask a similar question with the additional condition that the (absolutely simple) generic abelian variety should be equipped with non-trivial endomorphisms. (Such endomorphism structures then necessarily and uniquely extend to the whole semi-abelian scheme; see [19, IX, 1.4], [7, Ch. I, Proposition 2.7], or [14, Proposition 3.3.1.5].) We will also answer this question, with an affirmative answer similar to Theorem 1.3 (except in two special cases).

We note, however, that Question 1.2 and its analogue as in the last paragraph are not about the fibers of the tautological semi-abelian schemes carried by the toroidal compactifications of the Siegel moduli stacks or more general PEL moduli problems constructed in [7, Ch. IV] and [14, Ch. 6], because only split tori are used (and needed in the valuative criterion for properness) in the constructions there. Nevertheless, our use of the theory of degeneration is inspired by such constructions.

Here is an outline of the article. In $\S 2$, we construct explicit families for one-dimensional tori. In $\S 3$, we review some basic facts about the descent data for tori, and give the above-mentioned explanation why Question 1.1 is a bit too naive. To attack Question 1.2 in general, we recall relevant facts from the theory of degeneration in $\$ 4$. Using this theory, we give the proof of Theorem 1.3 in $\$ \S 5$ and 6. Finally, in $\$ 7$, we turn to the conditions for tori to be realizable as the degeneration of absolutely simple abelian varieties with nontrivial endomorphisms.

$\S 2$. One-dimensional case. Let $T$ be an algebraic torus of dimension one over a field $k$. Let us fix the choice of a separable closure $k^{s}$ of $k$. Then the action of the Galois group $\operatorname{Gal}\left(k^{s} / k\right)$ on the character group $X^{*}(T) \cong \mathbb{Z}$ of $T$ is either trivial (in which case $T$ is split), or surjects onto $\{ \pm 1\}$, corresponding to a separable quadratic extension $\widetilde{k}$ of $k$ in $k^{s}$. 
Let $R$ be any Dedekind domain and $\mathfrak{P}=(\pi)$ a principal maximal ideal of $R$ with residue field $k$. (For example, $R=k[t]$ and $\mathfrak{P}=(t)$.)

Proposition 2.1. Suppose that $\operatorname{char}(k) \neq 2$, and write $\tilde{k}=k(\sqrt{b})$, where $b \in k$ is a non-square element. Then for any lift $\mathfrak{b} \in R$ of $b$, the Weierstra $\beta$ equation

$$
Y^{2} Z=X^{3}+\mathfrak{b} X^{2} Z+\pi Z^{3}
$$

over $\operatorname{Spec}(R)$ defines an elliptic curve over the fraction field of $R$, has semistable reduction at $\mathfrak{P}$, and the fiber of the Néron model at $\mathfrak{P}$ is the $k$-torus on whose character group $\mathrm{Gal}\left(k^{s} / k\right)$ acts via the quadratic character of $\widetilde{k} / k$.

Proof. The discriminant of the Weierstraß equation (see [21, p. 180]) is given by

$$
\Delta=(-16 \pi)\left(4 \mathfrak{b}^{3}+27 \pi\right)
$$

This is non-zero, and is in fact a uniformizer, in $R_{\mathfrak{P}}$, since 2 is a unit in $R_{\mathfrak{P}}$; hence the first assertion.

Let $C$ denote the singular curve at $\mathfrak{P}$ :

$$
Y^{2} Z=X^{3}+b X^{2} Z \text {. }
$$

The complement of the unique singular point $P=[0: 0: 1]$ in $C$ is the fiber of the Néron model. We know that it is a one-dimensional torus, and that it splits over $\widetilde{k}$. Therefore, it is enough to show that it is not split over $k$.

Let $\pi: \widetilde{C} \rightarrow C$ be the blowup of $C$ at $P$, which induces an isomorphism $C \backslash\{P\} \cong \widetilde{C} \backslash \pi^{-1}(P)$. Because the slopes of the tangent cone

$$
y^{2}=b x^{2}
$$

to $C$ at $P$ are $\pm \sqrt{b}$, which are not contained in $k$, the inverse image $\pi^{-1}(P)$ consists of one point of degree 2 on $\widetilde{C}$. If $C \backslash\{P\}$ were split over $k$, this would not happen.

Proposition 2.2. Suppose that $\operatorname{char}(k)=2$, and $\widetilde{k}$ be a separable quadratic extension of $k$. Write $\vec{k}$ as the Artin-Schreier extension $\widetilde{k}=k(\alpha)$, where

$$
\alpha^{2}+\alpha+b=0
$$

and $b \in k$. Then for any lift $\mathfrak{b} \in R$ of $b$, the Weierstraß equation

$$
Y^{2} Z+X Y Z=X^{3}+\mathfrak{b} X^{2} Z+\pi Z^{3}
$$

over $\operatorname{Spec}(R)$ defines an elliptic curve over the fraction field of $R$, has semistable reduction at $\mathfrak{P}$, and the fiber of the Néron model at $\mathfrak{P}$ is the $k$-torus on whose character group $\operatorname{Gal}\left(k^{s} / k\right)$ acts via the quadratic character of $\tilde{k} / k$.

Proof. The proof is parallel. We only note that the discriminant

$$
\Delta=-(1+4 \mathfrak{b})^{3} \pi-16 \cdot 27 \pi^{2}
$$

is again a uniformizer in $R_{\mathfrak{P}}$, this time because 2 is in $\mathfrak{P}$. 
§3. Descent data for tori. In this section, we review some basic facts about the descent data for tori. At the end of this section, we explain that the rather naive Question 1.1 can be easily reduced to the case of $\mathbb{G}_{m}$, which can then be answered in the affirmative by the classical theory of Tate curves. In fact, the argument will also show that the construction of degenerating abelian varieties can be made functorial (and compatible with base field extensions) in the tori.

We start with the following basic fact.

LEMMA 3.1. Let $R$ be a complete noetherian local ring with residue field $k$. Then pulling back from $R$ to $k$ induces an equivalence from the category of isotrivial tori over $R$ to the category of tori over $k$. (Recall that a torus is isotrivial if it splits over a finite étale cover of its base scheme; see [6, IX, 1.1].)

Proof. This follows from [6, X, 3.3]. (Indeed, by [6, X, 2.1], all tori over $s=\operatorname{Spec}(k)$ are isotrivial, and so the lemma is a consequence of $[6, \mathrm{X}, 3.2]$.)

Now, suppose $R$ is any complete discrete valuation ring with residue field $k$ and fraction field $K$. Let $S:=\operatorname{Spec}(R), \eta:=\operatorname{Spec}(K)$, and $s:=\operatorname{Spec}(k)$. We shall denote the pullbacks to $\eta$ or $s$ by subscripts $\eta$ or $s$, respectively.

Suppose $T$ is an isotrivial torus over $S$. Then there exists a finite étale extension $\widetilde{R}$ of $R$, which we may and we shall assume to be a complete discrete valuation ring as well, such that $T_{\widetilde{S}}:=T \otimes_{R} \widetilde{R}$ is a split torus over $\widetilde{S}:=\operatorname{Spec}(\widetilde{R})$. Let $\widetilde{K}$ and $\widetilde{k}$ denote the fraction and residue fields of $\widetilde{R}$, respectively, and let $\widetilde{\eta}:=\operatorname{Spec}(\widetilde{K})$ and $\widetilde{s}:=\operatorname{Spec}(\widetilde{k})$. By $[8, \mathrm{~V}, 7$, and $4(\mathrm{~g})]$, up to replacing $\widetilde{R}$ with a further finite étale extension, we may and we shall assume that $\widetilde{K}$ is a Galois extension of $K$. Again, we shall denote the pullbacks to $\tilde{\eta}$ or $\widetilde{s}$ by subscripts $\tilde{\eta}$ or $\tilde{s}$, respectively.

Let $\underline{X}$ denote the character group of $T$, which is an étale sheaf of free abelian groups of finite ranks over $S$. Then $\underline{X}$ is the unique étale sheaf lifting $\underline{X}_{s}$, the character group of $T_{s}$. Let $n$ denote the relative dimension of $T$ over $S$. Then there exists some isomorphism $\xi: \mathbb{Z}^{n} \stackrel{\sim}{\rightarrow} \underline{X}_{\tilde{\eta}}$, where we abusively denote by $\mathbb{Z}^{n}$ the associated constant étale sheaf over $\tilde{\eta}$, which induces a representation $\rho: \operatorname{Gal}(\widetilde{K} / K) \rightarrow \mathrm{GL}_{n}(\mathbb{Z})$ giving the descent data for the étale sheaf $\underline{X}_{\tilde{\eta}}$. Since $\widetilde{R}$ is finite étale over $R$, we have $\operatorname{Gal}(\widetilde{K} / K) \cong \operatorname{Gal}(\widetilde{k} / k)$, and so $\rho$ determines and is determined by a representation $\operatorname{Gal}(\widetilde{k} / k) \rightarrow \mathrm{GL}_{n}(\mathbb{Z})$. Since the automorphism group of any torus is canonically isomorphic to the automorphism group of the associated character group, the representation $\rho$ also defines the data for $T_{S}$ (respectively $T_{s}$ ) to descend from $T_{\widetilde{S}} \cong \mathbb{G}_{\mathrm{m}, \widetilde{S}}^{n}$ (respectively $T_{\widetilde{S}} \cong \mathbb{G}_{\mathrm{m}, \widetilde{s}}^{n}$ ). (See, for example, [3, Ch. 6, §6.2, Example B] for an explanation that descent data for schemes with respect to the finite étale base extension $R \rightarrow \widetilde{R}$ as above are equivalent to actions of the Galois group $\operatorname{Gal}(\widetilde{K} / K)$ on the schemes.)

Conversely, any torus $T_{S}$ with character group $\underline{X}_{S}$ over $s$ canonically lifts to an isotrivial torus $T$ with character group $\underline{X}$ over $S$, by Lemma 3.1. If $T_{S}$ is of dimension $n$, then we have trivializations $\xi: \mathbb{Z}^{n} \stackrel{\sim}{\rightarrow} \underline{X}_{\tilde{\eta}}$ and representations $\rho: \operatorname{Gal}(\widetilde{K} / K) \cong \operatorname{Gal}(\tilde{k} / k) \rightarrow \mathrm{GL}_{n}(\mathbb{Z})$ as above, for suitable choices of $\widetilde{S}$, etc. 
PROPOSITION 3.2. Suppose $G_{0}$ is a semi-abelian scheme over $S$ with special fiber $G_{0, s} \cong \mathbb{G}_{\mathrm{m}, s}$ and with generic fiber $G_{0, \eta}$ an elliptic curve. Suppose $T_{s}$ is a torus over s, and suppose $\widetilde{S}=\operatorname{Spec}(\widetilde{R})$, etc, are as above. Then there exists a semi-abelian scheme $G$ over $S$ such that $G_{s} \cong T_{s}$, such that $G_{\eta}$ is an abelian variety, and such that $G_{\tilde{\eta}} \cong G_{0, \tilde{\eta}}^{n}$. In particular, Question 1.1 has an affirmative answer. Moreover, for a fixed choice of $G_{0}$, the assignment of $G$ to $T_{s}$ is functorial (and compatible with base field extensions) in the torus $T_{s}$.

Proof. By [19, XI, 1.13] (see also [14, Remark 3.3.3.9]), any semi-abelian scheme over the noetherian normal base scheme $S$ is equipped with some relative ample invertible sheaf over $S$. By the theory of fpqc descent (as in [8, VIII, 7.8]), all descent data for such quasi-projective semi-abelian schemes are effective. Since the representation $\rho: \operatorname{Gal}(\widetilde{K} / K) \cong \operatorname{Gal}(\widetilde{k} / k) \rightarrow \mathrm{GL}_{n}(\mathbb{Z})$ defining the data for $T_{S}$ to descend from $T_{\widetilde{S}} \cong \mathbb{G}_{\mathrm{m}, \widetilde{S}}^{n}$ also defines some descent data for $G_{0, \tilde{S}}^{n}$, which are effective as we have just explained, $G_{0, \widetilde{S}}^{n}$ descends to a uniquely determined semi-abelian scheme $G$ over $S$, whose generic fiber $G_{\eta}$ is an abelian scheme because $G_{\tilde{\eta}} \cong G_{0, \widetilde{\eta}}^{n}$ is; and whose special fiber $G_{s}$ is canonically isomorphic to $T_{s}$ because their descent data are both given by $\rho$. Such an assignment of $G$ to $T_{s}$ is functorial (and compatible with base field extensions) in the torus $T_{S}$ because the construction of $G$ by descent depends only on the descent data for $T_{s}$ and on the choice of $G_{0}$.

Thus, we have reduced the more naive Question 1.1 to the case of the one-dimensional split torus $\mathbb{G}_{\mathrm{m}}$ (over any base field), which can then be answered by the classical theory of Tate curves (see, for example, the rather universal construction over $\mathbb{Z}[[q]]$ in $[\mathbf{5}$, Ch. VII]). However, any $G$ given by Proposition 3.2 (or any assignment that is functorial and compatible with base field extensions in $T_{s}$ ) must satisfy $G_{\tilde{\eta}} \cong G_{0, \tilde{\eta}}^{n}$ over some extension $\tilde{\eta}$ of $\eta$, which cannot be absolutely simple when $n>1$, and hence cannot be used to answer Question 1.2. We need a theory more general than that of Tate curves, which we shall review in the following section.

$\S 4$. Theory of degeneration. Let $R$ be a complete discrete valuation ring with residue field $k$ and fraction field $K$. Let $S:=\operatorname{Spec}(R), \eta:=\operatorname{Spec}(K)$, and $s:=$ $\operatorname{Spec}(k)$. We shall denote the pullbacks to $\eta$ or $s$ by subscripts $\eta$ or $s$, respectively.

Definition 4.1 (Cf. [14, Definition 4.4.2]). With the setting as above, the category $\operatorname{DEG}_{\text {pol }}^{\text {tor }}(R)$ has objects consisting of pairs $(G, \lambda)$ over $S=\operatorname{Spec}(R)$, where the following hold.

(1) The first entry $G$ is a semi-abelian scheme over $S$.

(2) The pullback $G_{\eta}$ is an abelian scheme over $\eta$, in which case there is a unique semi-abelian scheme $G^{\vee}$ (up to unique isomorphism) over $S$, called the dual semi-abelian scheme of $G$, such that $G_{\eta}^{\vee}$ is the dual abelian scheme of $G_{\eta}$ (see [14, §3.4.3]). 
(3) The pullback $G_{s}$ is a torus over $s$ (in which case $G_{s}^{\vee}$ also is).

(4) The second entry $\lambda: G \rightarrow G^{\vee}$ is a group homomorphism that induces by restriction a polarization $\lambda_{\eta}$ of $G_{\eta}$.

Remark 4.2. The definition of pairs $(G, \lambda)$ as in Definition 4.1 extends verbatim to the case where $S$ is a noetherian normal local scheme, but we stated the definition this way only because the theory of degeneration below requires $R$ to be complete, and we only need the special case over Dedekind domains.

Remark 4.3. By [19, XI, 1.13, and IX, 1.4] (see also [14, Remarks 3.3.3.9 and 4.4.3, and Proposition 3.3.1.5]), any semi-abelian scheme $G$ over a noetherian normal domain $R$ that is generically an abelian variety is automatically equipped with a homomorphism $\lambda: G \rightarrow G^{\vee}$ that is generically a polarization (of an abelian variety). In particular, any semi-abelian scheme $G$ over $S$ satisfying the first two conditions in Definition 4.1 is automatically equipped with some homomorphism $\lambda: G \rightarrow G^{\vee}$ satisfying the last condition in Definition 4.1.

By the theory of degeneration data (see [18]; see also [7, Chs II and III] and [14, Ch. 4; see, in particular, Corollary 4.5.4.31], with all abelian parts in the degenerations suppressed; or see, for example, [16, §2.7], for the corresponding rigid analytic theory, which is in some sense simpler), there is an equivalence of categories

$$
\mathrm{M}_{\mathrm{pol}}^{\mathrm{tor}}(R): \mathrm{DD}_{\mathrm{pol}}^{\mathrm{tor}}(R) \rightarrow \mathrm{DEG}_{\mathrm{pol}}^{\mathrm{tor}}(R):(\underline{X}, \underline{Y}, \phi, \tau) \mapsto(G, \lambda)
$$

realizing $(G, \lambda)$ (up to isomorphism) as the image of an object in $\operatorname{DD}_{\text {pol }}^{\text {tor }}(R)$ given by the following data.

(1) Two étale sheaves $\underline{X}$ and $\underline{Y}$ of free abelian groups of finite ranks over $S$, together with an embedding $\phi: \underline{Y} \rightarrow \underline{X}$ with finite cokernel (here $\phi: \underline{Y} \rightarrow$ $\underline{X}$ corresponds to the isogeny of tori $T \rightarrow T^{\vee}$ uniquely lifting $G_{s} \rightarrow G_{s}^{\vee}$, by Lemma 3.1. It should be noted that the notation for objects related to the degeneration of $G_{\eta}^{\vee}$, such as $T^{\vee}$, is only symbolic in nature).

(2) A bimultiplicative homomorphism $\tau: \underline{Y}_{\eta} \times \underline{X}_{\eta} \rightarrow \mathbb{G}_{\mathrm{m}, \eta}$ of étale sheaves over $\eta$ with symmetric pullback under $\operatorname{Id}_{\underline{Y}} \times \underline{\phi}: \underline{Y} \times \underline{Y} \rightarrow \underline{Y} \times \underline{X}$, satisfying the following positivity condition for some (and hence every) finite extension $\widetilde{K}$ of $K$ as in $\S 3$ over which both $\underline{X}_{\widetilde{\eta}}$ and $\underline{Y}_{\widetilde{\eta}}$, where $\tilde{\eta}:=\operatorname{Spec}(\tilde{K})$, are constant: $\widetilde{v}(\tau(y, \phi(y)))>0$ for every non-zero section $y$ of $\underline{Y}_{\tilde{\eta}}$, where $\widetilde{v}: \widetilde{K}^{\times} \rightarrow \mathbb{Z}$ is any non-trivial discrete valuation of $\widetilde{K}$.

We say that $(\underline{X}, \underline{Y}, \phi, \tau)$ is the degeneration data of $(G, \lambda)$.

Remark 4.4. The bimultiplicative homomorphism $\tau: \underline{Y}_{\eta} \times \underline{X}_{\eta} \rightarrow \mathbb{G}_{\mathrm{m}, \eta}$ induces (by the definition of $\underline{X}$ as the character group of $T$ ) a homomorphism

$$
\iota: \underline{Y}_{\eta} \rightarrow T_{\eta},
$$

which is a generalization of the familiar Tate periods in the case of relative dimension one (i.e. of Tate curves). Nevertheless, we will not need $\iota$ in what follows. 
§. Conditions for degenerations. Let $R, k, K, S, s$, and $\eta$ be as in the beginning of $\S 4$.

Suppose that we have tori $T_{s}$ and $T_{s}^{\vee}$ over $s=\operatorname{Spec}(k)$ with character groups $\underline{X}_{s}$ and $\underline{Y}_{s}$ that are étale sheaves of free abelian groups of finite ranks, together with an embedding $\phi_{s}: \underline{Y}_{s} \hookrightarrow \underline{X}_{s}$ with finite cokernel inducing an isogeny $\lambda_{T_{s}}: T_{s} \rightarrow T_{s}^{\vee}$. (Certainly, we allow $\phi_{s}$ and hence $\lambda_{T_{s}}$ to be isomorphisms.) In this section, we shall find necessary and sufficient conditions for the existence of an object $(G, \lambda)$ in $\operatorname{DEG}_{\text {pol }}^{\text {tor }}(R)$ such that $\lambda_{s}: G_{s} \rightarrow G_{s}^{\vee}$ can be identified with $\lambda_{T_{s}}: T_{s} \rightarrow T_{s}^{\vee}$ (via some isomorphisms $G_{s} \cong T_{s}$ and $G_{s}^{\vee} \cong T_{s}^{\vee}$ over $s$ ), and such that $G_{\eta}$ and hence $G_{\eta}^{\vee}$ are absolutely simple abelian varieties.

By Lemma 3.1, the embedding $\phi_{s}: \underline{Y}_{s} \hookrightarrow \underline{X}_{s}$ lifts to an embedding $\phi: \underline{Y} \hookrightarrow$ $\underline{X}$ with finite cokernel over $S$ of étale sheaves of free abelian groups of finite ranks, inducing an isogeny $\lambda_{T}: T \rightarrow T^{\vee}$ (between isotrivial tori) lifting $\lambda_{T_{S}}$. Let $\widetilde{S}$, etc, be as in $\S 3$ such that both $\underline{X}_{\widetilde{\eta}}$ and $\underline{Y}_{\tilde{\eta}}$ are constant of some common finite rank $n$, so that there are isomorphisms $\xi: \mathbb{Z}^{n} \stackrel{\sim}{\rightarrow} \underline{X}_{\tilde{\eta}}$ and $\xi^{\vee}: \mathbb{Z}^{n} \stackrel{\sim}{\rightarrow} \underline{Y}_{\tilde{\eta}}$ and representations $\rho: \operatorname{Gal}(\widetilde{K} / K) \rightarrow \operatorname{GL}_{n}(\mathbb{Z})$ and $\rho^{\vee}: \operatorname{Gal}(\widetilde{K} / K) \rightarrow \operatorname{GL}_{n}(\mathbb{Z})$ defining the descent data for the étale sheaves $\underline{X}_{\widetilde{\eta}}$ and $\underline{Y}_{\tilde{\eta}}$. Consider

$$
\phi_{\xi, \xi^{\vee}}:=\xi^{-1} \phi_{\widetilde{\eta}} \xi^{\vee}: \mathbb{Z}^{n} \rightarrow \mathbb{Z}^{n}
$$

which defines an element of $\operatorname{End}\left(\mathbb{Z}^{n}\right) \cap \operatorname{Aut}\left(\mathbb{Q}^{n}\right) \cong \mathrm{M}_{n}(\mathbb{Z}) \cap \mathrm{GL}_{n}(\mathbb{Q})$.

LEMMA 5.2. We have

$$
\phi_{\xi, \xi^{\vee}}\left(\rho^{\vee}(\gamma) z\right)=\rho(\gamma)\left(\phi_{\xi, \xi^{\vee}}(z)\right)
$$

for all $\gamma \in \operatorname{Gal}(\widetilde{K} / K)$ and $z \in \mathbb{Z}^{n}$.

Proof. This is because $\phi: \underline{Y} \rightarrow \underline{X}$ is a morphism defined over $S$.

Proposition 5.3. With the setting as above, with the fixed choices of some $\xi, \xi^{\vee}, \rho$, and $\rho^{\vee}$, the datum of an object $(G, \lambda)$ of $\operatorname{DEG}_{\mathrm{pol}}^{\mathrm{tor}}(R)$ (as in Definition 4.1) such that $\lambda_{s}: G_{s} \rightarrow G_{s}^{\vee}$ can be identified with $\lambda_{T_{s}}: T_{s} \rightarrow T_{s}^{\vee}$ (via some isomorphisms $G_{s} \cong T_{s}$ and $G_{s}^{\vee} \cong T_{s}^{\vee}$ over $s$ ) is equivalent to the datum of a bimultiplicative pairing

$$
\langle\cdot, \cdot\rangle_{\tau, \xi, \xi^{\vee}}: \mathbb{Z}^{n} \times \mathbb{Z}^{n} \rightarrow \widetilde{K}^{\times}
$$

satisfying the following conditions.

(1) (Galois equivariance) $\left\langle\rho^{\vee}(\gamma) z, \rho(\gamma) w\right\rangle_{\tau, \xi, \xi^{\vee}}=\gamma\langle z, w\rangle_{\tau, \xi, \xi^{\vee}}$ for all $z$, $w \in \mathbb{Z}^{n}$ and $\gamma \in \operatorname{Gal}(\widetilde{K} / K)$ (note that the action of $\operatorname{Gal}(\widetilde{K} / K)$ on $\widetilde{K}^{\times}$ here is the naive one, because $\widetilde{K}^{\times}$is just the group of points of $\mathbb{G}_{\mathrm{m}}$ over $\tilde{\eta}=\operatorname{Spec}(\widetilde{K})$, and $\mathbb{G}_{\mathrm{m}}$ is already defined over $\eta=\operatorname{Spec}(K)$ or rather $\operatorname{Spec}(\mathbb{Z}))$.

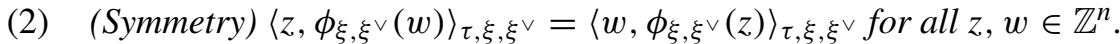

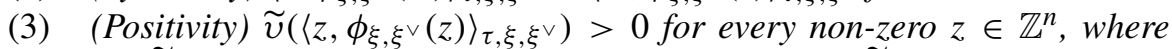
$\widetilde{v}: \widetilde{K}^{\times} \rightarrow \mathbb{Z}$ is any non-trivial discrete valuation of $\widetilde{K}$. 
Proof. This follows from the theory reviewed in $\S 4$, by base change to $\tilde{\eta}$, by pulling back under $\xi^{\vee} \times \xi$, and by descent (as in the proof of Proposition 3.2).

Proposition 5.5. In the setting of Proposition 5.3, if the pairing $\langle\cdot, \cdot\rangle_{\tau, \xi, \xi^{\vee}}$ corresponding to $(G, \lambda)$ satisfies the additional condition that

$$
\langle z, w\rangle_{\tau, \xi, \xi^{\vee}} \neq 1
$$

for all non-zero $z, w \in \mathbb{Z}^{n}$, then $G_{\eta}$ and hence $G_{\eta}^{\vee}$ must be absolutely simple.

Proof. Suppose $G_{\eta}$ is not absolutely simple. Then there exists a finite extension $K^{\prime}$ of $\widetilde{K}$ over which the base change of $G_{\eta}$ is isogenous to a nontrivial product of two abelian varieties of smaller dimension. By the theory of Néron models (see, in particular, [3, Ch. 7, §7.4, Theorem 1]), up to replacing $K^{\prime}$ with a finite extension, we may assume that these two abelian varieties extend to two semi-abelian schemes $G_{1}$ and $G_{2}$, respectively, over the integral closure $R^{\prime}$ of $R$ in $K^{\prime}$. Since the theory of degeneration in $\S 4$ is an equivalence of categories, there exist some non-zero $z, w \in \mathbb{Z}^{n}$, which are the images of some elements of the character groups of the torus parts of $G_{1}^{\vee}$ and $G_{2}$, such that $\langle z, w\rangle_{\tau, \xi, \xi^{\vee}}=1$ (in $K^{\prime}$, and hence also in $\widetilde{K}$ ). This contradicts the condition (5.6) in this proposition, as desired.

In the remainder of this section, let us show that the conditions in Propositions 5.3 and 5.5 can indeed be achieved by some bimultiplicative pairing $\langle\cdot, \cdot\rangle_{\tau, \xi, \xi^{\vee}}$.

LEMMA 5.7. There exists $\theta \in \widetilde{R}^{\times}$such that $\{\gamma \theta\}_{\gamma \in \operatorname{Gal}(\widetilde{K} / K)}$ form a free $R$ basis of $\widetilde{R}$, and such that the reductions $\{\gamma \bar{\theta}\}_{\gamma \in \operatorname{Gal}(\widetilde{K} / K)}$ in $\widetilde{k}$ form a $k$-basis of $\widetilde{k}$.

Proof. Since $\operatorname{Gal}(\widetilde{K} / K)$ is a finite group, the group algebra $R[\operatorname{Gal}(\widetilde{K} / K)]$ over $R$ is an $R$-order (see [14, Definition 1.1.1.3]) in the finite-dimensional group algebra $K[\operatorname{Gal}(\widetilde{K} / K)]$ over $K$. Since $\widetilde{R}$ is finite étale over $R$, we have $\operatorname{Gal}(\widetilde{K} / K) \cong \operatorname{Gal}(\widetilde{k} / k)$ as $\operatorname{groups}$, and $R[\operatorname{Gal}(\widetilde{K} / K)] \otimes_{R} k \cong k[\operatorname{Gal}(\tilde{k} / k)]$ as group algebras over $k$. By applying the normal basis theorem (see, for example, [15, Ch. VI, §13]) to the finite Galois field extension $\widetilde{k} / k$, we have $\widetilde{k} \cong k[\operatorname{Gal}(\tilde{k} / k)]$ as left $k[\operatorname{Gal}(\tilde{k} / k)]$-modules. Therefore, since $R$ is local with residue field $k$, by [14, Lemma 1.1.3.1] (which is a consequence of the usual Nakayama's lemma for finitely generated $R$-modules), we have $\widetilde{R} \cong$ $R[\operatorname{Gal}(\widetilde{K} / K)]$ as left $R[\operatorname{Gal}(\widetilde{K} / K)]$-modules, and the lemma follows.

LEMMA 5.8. There exists $u_{0} \in \widetilde{R}^{\times}$such that the Galois conjugates $\left\{\gamma u_{0}\right\}_{\gamma \in \operatorname{Gal}(\widetilde{K} / K)}$ are multiplicatively independent in the sense that, if

$$
\prod_{\gamma \in \operatorname{Gal}(\widetilde{K} / K)}\left(\gamma u_{0}\right)^{c_{\gamma}}=1
$$

in $\widetilde{R}^{\times}$for some integers $\left\{c_{\gamma}\right\}_{\gamma \in \operatorname{Gal}(\widetilde{K} / K)}$, then these integers are all zero. 
Proof. Let $I$ and $\widetilde{I}$ denote the maximal ideals of $R$ and $\widetilde{R}$, respectively, which satisfy $\widetilde{I}=I \cdot \widetilde{R}$ because $\widetilde{R}$ is finite étale over $R$. If $\operatorname{char}(k)=0$, we set $\delta=0$. Otherwise, we have $\operatorname{char}(k)=p$ for some rational prime number $p>0$, and we fix the choice of any integer $\delta \geqslant 0$ such that the multiplicative subgroup $1+\widetilde{I}^{1+\delta}$ of $\widetilde{R}^{\times}$does not contain any non-trivial $p$ th roots of unity. (Such an integer $\delta$ always exists, because there are only finitely many $p$ th root of unity in $\widetilde{K}$ or any field, and because $\widetilde{R}$ is $\widetilde{I}$-adically separated.)

Let $\theta \in \widetilde{R}^{\times}$be as in Lemma 5.7, let $\varpi$ be any uniformizer of $R$ (which is then also a uniformizer of $\widetilde{R}$ ), let $\delta$ be as in the previous paragraph, and let

$$
u_{0}:=1+\theta \varpi^{1+\delta} \in 1+\widetilde{I}^{1+\delta} \subset \widetilde{R}^{\times} .
$$

We would like to show that $u_{0}$ satisfies the requirement of this lemma; namely, if

$$
\prod_{\gamma \in \operatorname{Gal}(\widetilde{K} / K)}\left(\gamma u_{0}\right)^{c_{\gamma}}=\prod_{\gamma \in \operatorname{Gal}(\widetilde{K} / K)}\left(1+(\gamma \theta) \varpi^{1+\delta}\right)^{c_{\gamma}}=1
$$

in $1+\widetilde{I}^{1+\delta} \subset \widetilde{R}^{\times}$for some integers $\left\{c_{\gamma}\right\}_{\gamma \in \operatorname{Gal}(\widetilde{K} / K)}$, then these integers are all zero.

Suppose, to the contrary, that the relation (5.9) holds for some integers $\left\{c_{\gamma}\right\}_{\gamma \in \operatorname{Gal}(\widetilde{K} / K)}$ that are not all zero. First, suppose $\operatorname{char}(k)=0$. Then $\mathbb{Q} \subset k$, and the relation (5.9) implies that

$$
\sum_{\gamma \in \operatorname{Gal}(\widetilde{K} / K)} c_{\gamma}(\gamma \bar{\theta})=0
$$

in $k$, by considering the images of the terms in (5.9) in $\left(1+\widetilde{I}^{1+\delta}\right) /\left(1+\widetilde{I}^{2+\delta}\right) \cong$ $k$ (where $\delta=0)$. This contradicts the linear independence of $\{\gamma \bar{\theta}\}_{\gamma \in \operatorname{Gal}(\widetilde{K} / K)}$ over $k$. Otherwise, suppose $\operatorname{char}(k)=p>0$. Then there exists some power $q$ of $p$ such that $q \mid c_{\gamma}$ for all $\gamma \in \operatorname{Gal}(\widetilde{K} / K)$, but $(p q) \nmid c_{\gamma_{0}}$ for at least one $\gamma_{0} \in \operatorname{Gal}(\widetilde{K} / K)$, so that the reductions $\left\{\bar{c}_{\gamma}:=\left(c_{\gamma} / q\right) \bmod p\right\}$ are not all zero in $\mathbb{F}_{p} \subset k$. By the choice of $\delta$, the multiplicative subgroup $1+\widetilde{I}^{1+\delta}$ of $\widetilde{R}^{\times}$cannot contain any non-trivial $p$ th roots of unity, and consequently the relation (5.9) still holds with the $c_{\gamma}$ replaced with $c_{\gamma} / q$. After such a replacement, again by considering the images of the terms in $(5.9)$ in $\left(1+\widetilde{I}^{1+\delta}\right) /\left(1+\widetilde{I}^{2+\delta}\right) \cong k$, we get that

$$
\sum_{\gamma \in \operatorname{Gal}(\widetilde{K} / K)} \bar{c}_{\gamma}(\gamma \bar{\theta})=0
$$

in $k$. This again contradicts the linear independence of $\{\gamma \bar{\theta}\}_{\gamma \in \operatorname{Gal}(\widetilde{K} / K)}$ over $k$. Thus, the integers $\left\{c_{\gamma}\right\}_{\gamma \in \operatorname{Gal}(\widetilde{K} / K)}$ must be all zero, and the lemma follows.

LEMMA 5.10. For any integer $N \geqslant 1$, there exist elements $u_{1}, u_{2}, \ldots, u_{N}$ in $\widetilde{R}^{\times}$that are multiplicatively Galois independent in the sense that, if

$$
\prod_{1 \leqslant i \leqslant N ; \gamma \in \operatorname{Gal}(\tilde{K} / K)}\left(\gamma u_{i}\right)^{c_{i, \gamma}}=1
$$

in $\widetilde{R}^{\times}$for some integers $\left\{c_{i, \gamma}\right\}_{1 \leqslant i \leqslant N ; \gamma \in \operatorname{Gal}(\widetilde{K} / K)}$, then these integers are all zero. 
Proof. Let $u_{0} \in \widetilde{R}^{\times}$be as in Lemma 5.8, and let $\varpi$ be a uniformizer of $R$ (which does not have to be the same as the uniformizer in the proof of Lemma 5.8). Let us choose elements $t_{1}, t_{2}, \ldots, t_{N}$ in $K^{\times}$that are algebraically independent over the subfield $\widetilde{K}_{0}$ of $\widetilde{K}$ generated by $\varpi$ and the finite subset $\left\{\gamma u_{0}\right\}_{\gamma \in \operatorname{Gal}(\widetilde{K} / K)}$ over its prime subfield. This is possible because the transcendence degree of $\widetilde{K}$ over its prime subfield is (uncountably) infinite. For each $1 \leqslant i \leqslant N$, up to replacing $t_{i}$ with a suitable multiple by a power of $\varpi$, we may assume that $t_{i} \in R^{\times}$. Let

$$
u_{i}:=1+u_{0} t_{i} \varpi \in \widetilde{R}^{\times}
$$

so that

$$
\gamma u_{i}=1+\left(\gamma u_{0}\right) t_{i} \varpi \in \widetilde{R}^{\times},
$$

for each $1 \leqslant i \leqslant N$. We would like to show that these elements $\left\{u_{i}\right\}_{1 \leqslant i \leqslant N}$ satisfy the requirement of the lemma; namely, if

$$
\prod_{1 \leqslant i \leqslant N ; \gamma \in \operatorname{Gal}(\widetilde{K} / K)}\left(\gamma u_{i}\right)^{c_{i, \gamma}}=\prod_{1 \leqslant i \leqslant N ; \gamma \in \operatorname{Gal}(\widetilde{K} / K)}\left(1+\left(\gamma u_{0}\right) t_{i} \varpi\right)^{c_{i, \gamma}}=1
$$

for some integers $\left\{c_{i, \gamma}\right\}_{1 \leqslant i \leqslant N ; \gamma \in \operatorname{Gal}(\widetilde{K} / K)}$, then these integers are all zero.

For all $i$ and $\gamma$, set $c_{i, \gamma}^{+}=c_{i, \gamma}$ and $c_{i, \gamma}^{-}=0$ when $c_{i, \gamma} \geqslant 0$, and set $c_{i, \gamma}^{+}=0$ and $c_{i, \gamma}^{-}=-c_{i, \gamma}$ when $c_{i, \gamma} \leqslant 0$. Then the relation (5.11) implies that

$$
\begin{gathered}
\prod_{1 \leqslant i \leqslant N ; \gamma \in \operatorname{Gal}(\widetilde{K} / K)}\left(1+\left(\gamma u_{0}\right) t_{i} \varpi\right)^{c_{i, \gamma}^{+}} \\
=\prod_{1 \leqslant i \leqslant N ; \gamma \in \operatorname{Gal}(\widetilde{K} / K)}\left(1+\left(\gamma u_{0}\right) t_{i} \varpi\right)^{c_{i, \gamma}^{-}} .
\end{gathered}
$$

Since $t_{1}, t_{2}, \ldots, t_{N}$ are algebraically independent over the subfield $\widetilde{K}_{0}$ of $\widetilde{K}$, the relation (5.12) is possible only when the corresponding "polynomials" in $t_{1}, t_{2}$, $\ldots, t_{N}$ (with coefficients in $\widetilde{K}_{0}$ ) on the two sides match. In particular, for each $1 \leqslant i \leqslant N$, by comparing the non-zero top degree "monomials" purely in $t_{i}$ (with coefficients in $\widetilde{K}_{0}$ ) on the two sides, we have (by comparing exponents)

$$
\sum_{\gamma \in \operatorname{Gal}(\widetilde{K} / K)} c_{i, \gamma}^{+}=\sum_{\gamma \in \operatorname{Gal}(\widetilde{K} / K)} c_{i, \gamma}^{-}
$$

in $\mathbb{Z}_{\geqslant 0}$, and have (by comparing coefficients, after canceling powers of $\varpi$ )

$$
\prod_{\gamma \in \operatorname{Gal}(\widetilde{K} / K)}\left(\gamma u_{0}\right)^{c_{i, \gamma}^{+}}=\prod_{\gamma \in \operatorname{Gal}(\widetilde{K} / K)}\left(\gamma u_{0}\right)^{c_{i, \gamma}^{-}}
$$

in $\widetilde{R}^{\times}$. By the assumption on $u_{0}$ (satisfying the requirement in Lemma 5.8), and by the definition of $c_{i, \gamma}^{+}$and $c_{i, \gamma}^{-}$, this last relation (5.13) forces all $c_{i, \gamma}^{+}, c_{i, \gamma}^{-}$, and $c_{i, \gamma}$ to be zero, for all $\gamma \in \operatorname{Gal}(\widetilde{K} / K)$, as desired.

PROPOSITION 5.14. There exists a bimultiplicative pairing

$$
\langle\cdot, \cdot\rangle_{\tau, \xi, \xi \vee}: \mathbb{Z}^{n} \times \mathbb{Z}^{n} \rightarrow \widetilde{K}^{\times}
$$

as in (5.4) satisfying all the conditions in Propositions 5.3 and 5.5. 
Proof. Let

$$
\langle\cdot, \cdot\rangle_{0}: \mathbb{Z}^{n} \times \mathbb{Z}^{n} \rightarrow \mathbb{Z}
$$

be any positive definite (symmetric) bilinear pairing. Let $e_{1}, \ldots, e_{n}$ denote the standard basis vectors of $\mathbb{Z}^{n}$, let $\varpi$ be any element of $K^{\times}$of positive valuation, and let $\left\{u_{i j}\right\}_{1 \leqslant i \leqslant j \leqslant n}$ be elements in $\widetilde{R}^{\times}$that are multiplicatively Galois independent as in Lemma 5.10. Then we define a symmetric bimultiplicative pairing

$$
\langle\cdot, \cdot\rangle_{1}: \mathbb{Z}^{n} \times \mathbb{Z}^{n} \rightarrow \widetilde{K}^{\times}
$$

by setting

$$
\left\langle e_{i}, e_{j}\right\rangle_{1}:=u_{i j} \cdot \varpi^{\left\langle e_{i}, e_{j}\right\rangle_{0}}
$$

for all $1 \leqslant i \leqslant j \leqslant n$, and by extending the values of the pairing to the whole domain $\mathbb{Z}^{n} \times \mathbb{Z}^{n}$ by symmetry and bimultiplicativity. Next, we define a symmetric bimultiplicative pairing

$$
\langle\cdot, \cdot\rangle_{2}: \mathbb{Z}^{n} \times \mathbb{Z}^{n} \rightarrow \widetilde{K}^{\times}
$$

satisfying the Galois equivariance

$$
\langle\rho(\gamma) z, \rho(\gamma) w\rangle_{2}=\gamma\langle z, w\rangle_{2}
$$

for all $\gamma \in \operatorname{Gal}(\widetilde{K} / K)$ and $z, w \in \mathbb{Z}^{n}$, by setting

$$
\langle z, w\rangle_{2}:=\prod_{\gamma \in \operatorname{Gal}(\widetilde{K} / K)} \gamma^{-1}\langle\rho(\gamma) z, \rho(\gamma) w\rangle_{1}
$$

for all $z, w \in \mathbb{Z}^{n}$. Finally, we define the desired bimultiplicative pairing

$$
\langle\cdot, \cdot\rangle_{\tau, \xi, \xi^{\vee}}: \mathbb{Z}^{n} \times \mathbb{Z}^{n} \rightarrow \widetilde{K}^{\times}
$$

by setting

$$
\langle z, w\rangle_{\tau, \xi, \xi^{\vee}}:=\left\langle\phi_{\xi, \xi^{\vee}}(z), w\right\rangle_{2}
$$

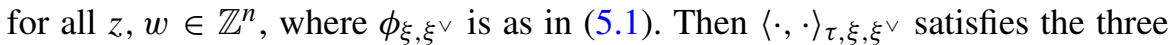
conditions (1), (2), and (3) in Proposition 5.3 by the symmetry and positive definiteness of $\langle\cdot, \cdot\rangle_{0}$; by the definitions of the pairings $\langle\cdot, \cdot\rangle_{1}$ and $\langle\cdot, \cdot\rangle_{2}$; by the choices of $\varpi$ (of positive valuation in $\widetilde{K}^{\times}$) and $\left\{u_{i j}\right\}_{1 \leqslant i \leqslant j \leqslant n}$ (of zero valuation in $\left.\widetilde{K}^{\times}\right)$; and by Lemma 5.2 and the relations (5.15) and (5.17).

It remains to show that $\langle z, w\rangle_{\tau, \xi, \xi^{\vee}} \neq 1$ (as in (5.6)) for all non-zero $z$, $w \in \mathbb{Z}^{n}$. Since $\phi_{\xi, \xi \vee}$ is an embedding, by the defining relation (5.17), it suffices to show that $\langle z, w\rangle_{2} \neq 1$ for all non-zero $z, w \in \mathbb{Z}^{n}$. By the choice of $\left\{u_{i j}\right\}_{1 \leqslant i \leqslant j \leqslant n}$, the terms in the product (5.16) indexed by different elements $\gamma$ have multiplicatively independent values (up to powers of $\varpi$ ), and so it suffices to show that $\langle z, w\rangle_{1} \neq 1$ for all non-zero $z, w \in \mathbb{Z}^{n}$. Suppose, to the contrary, that there are some $z=\sum_{1 \leqslant i \leqslant n} a_{i} e_{i}$ and $w=\sum_{1 \leqslant i \leqslant n} b_{i} e_{i}$, where $\left(a_{i}\right)_{1 \leqslant i \leqslant n}$ and $\left(b_{i}\right)_{1 \leqslant i \leqslant n}$ are non-zero $n$-tuples of integers, such that $\langle z, w\rangle_{1}=1$. Then we have

$$
\left(\prod_{1 \leqslant i \leqslant n}\left\langle e_{i}, e_{i}\right\rangle_{1}^{a_{i} b_{i}}\right) \cdot\left(\prod_{1 \leqslant i<j \leqslant n}\left\langle e_{i}, e_{j}\right\rangle_{1}^{a_{i} b_{j}+b_{i} a_{j}}\right)=1
$$


in $\widetilde{K}^{\times}$, which implies that

$$
\left(\prod_{1 \leqslant i \leqslant n} u_{i i}^{a_{i} b_{i}}\right) \cdot\left(\prod_{1 \leqslant i<j \leqslant n} u_{i j}^{a_{i} b_{j}+b_{i} a_{j}}\right)=1
$$

in $\widetilde{R}^{\times}$(by pulling out all powers of $\varpi$ ). By the choices of $\left\{u_{i j}\right\}_{1 \leqslant i \leqslant j \leqslant n}$ again, the identity (5.18) is possible only when

$$
a_{i} b_{j}+b_{i} a_{j}=0
$$

in $\mathbb{Z}$ for all $1 \leqslant i \leqslant j \leqslant n$. Let $i_{0}$ (respectively $j_{0}$ ) be the smallest index $i$ (respectively $j$ ) such that $a_{i} \neq 0$ (respectively $b_{j} \neq 0$ ), which exists by assumption. But then $a_{i_{0}} b_{j_{0}}+a_{j_{0}} b_{i_{0}} \neq 0$. This contradicts the condition (5.19), as desired.

Remark 5.20. If we start with any element $\varpi$ in $K^{\times}$of positive valuation and any positive definite (symmetric) bilinear pairing $\langle\cdot, \cdot\rangle_{0}: \mathbb{Z}^{n} \times \mathbb{Z}^{n} \rightarrow \mathbb{Z}$, and consider the Galois invariant pairing $\langle\cdot, \cdot\rangle_{1}: \mathbb{Z}^{n} \times \mathbb{Z}^{n} \rightarrow \mathbb{Z}$ defined by the sum

$$
\langle z, w\rangle_{1}:=\sum_{\gamma \in \operatorname{Gal}(\widetilde{K} / K)}\langle\rho(\gamma) z, \rho(\gamma) w\rangle_{0},
$$

then the bimultiplicative pairing $\langle\cdot, \cdot\rangle_{\tau, \xi, \xi^{\vee}}: \mathbb{Z}^{n} \times \mathbb{Z}^{n} \rightarrow \widetilde{K}^{\times}$defined by

$$
\langle z, w\rangle_{\tau, \xi, \xi^{\vee}}:=\varpi^{\left\langle\phi_{\xi, \xi \vee}(z), w\right\rangle_{1}} \in K^{\times}
$$

satisfies all the conditions in Proposition 5.3. However, by completion of squares, there exists $f \in \mathrm{GL}_{n}(\mathbb{Q})$ such that $(f \times f)^{*} \circ\left(\langle\cdot, \cdot\rangle_{1} \otimes_{\mathbb{Z}} \mathbb{Q}\right): \mathbb{Q}^{n} \times \mathbb{Q}^{n} \rightarrow$ $\mathbb{Q}$ decomposes as an orthogonal direct sum of pairings over one-dimensional subspaces. Thus, when $n>1$, no pairing $\langle\cdot, \cdot \cdot\rangle_{\tau, \xi, \xi}$ defined as above can possibly satisfy the condition in Proposition 5.5. Furthermore, by Proposition 5.3, and by the functoriality in the theory of degeneration in $\$ 4$, some non-zero multiple of $f$ defines an isogeny between $G_{\tilde{\eta}}$ and a product of one-dimensional abelian varieties (i.e. elliptic curves) over $\widetilde{\eta}$. In particular, when $n>1$, no $G_{\eta}$ thus obtained can be absolutely simple.

Remark 5.21. If we consider only $K^{\times}$-valued pairings

$$
\langle\cdot, \cdot\rangle_{\tau, \xi, \xi^{\vee}}: \mathbb{Z}^{n} \times \mathbb{Z}^{n} \rightarrow K^{\times}
$$

satisfying all the conditions in Proposition 5.3, then the Galois equivariance condition (1) there becomes the simpler Galois invariance condition

$$
\left\langle\rho^{\vee}(\gamma) z, \rho(\gamma) w\right\rangle_{\tau, \xi, \xi^{\vee}}=\langle z, w\rangle_{\tau, \xi, \xi^{\vee}}
$$

for all $z, w \in \mathbb{Z}^{n}$ and $\gamma \in \operatorname{Gal}(\tilde{K} / K)$. Since $\operatorname{char}(\mathbb{Q})=0$, and since $\operatorname{Gal}(\tilde{K} / K)$ is finite, the (finite-dimensional) $\mathbb{Q}$-subalgebra $B$ of $\operatorname{End}\left(\mathbb{Q}^{n}\right)$ generated by the images $\{\rho(\gamma)\}_{\gamma \in \operatorname{Gal}(\widetilde{K} / K)}$ issemisimple (see, for example, [4, Theorem 15.6 and 
(25.8)]), and the $\mathbb{Z}$-subalgebra $\mathcal{O}$ of $\operatorname{End}\left(\mathbb{Z}^{n}\right)$ generated by the same images is an order in $B$. The anti-automorphism $\gamma \mapsto \gamma^{-1}$ of $\operatorname{Gal}(\tilde{K} / K)$ induces an involution $\star: B \rightarrow B$ stabilizing $\mathcal{O}$, which is positive in the sense that $\operatorname{Tr}_{B / \mathbb{Q}}\left(b b^{\star}\right)>0$ for all non-zero $b \in B$. (Such orders and algebras with positive involutions are exactly the ones considered in the context of endomorphisms of abelian varieties; see $\$ 7$ below.) Then the above condition (5.22) can be rewritten as

$$
\langle b z, w\rangle_{\tau, \xi, \xi^{\vee}}=\left\langle z, b^{\star} w\right\rangle_{\tau, \xi, \xi^{\vee}}
$$

for all $z, w \in \mathbb{Z}^{n}$ and $b \in \mathcal{O}$. By Proposition 5.3, and by the functoriality in the theory of degeneration in $\S 4$, we obtain an injective homomorphism $i_{\tilde{S}}$ : $\mathcal{O} \rightarrow \operatorname{End}_{\widetilde{S}}\left(G_{\widetilde{S}}\right)$, whose restriction $i_{\tilde{\eta}}: \mathcal{O} \rightarrow \operatorname{End}_{\tilde{\eta}}\left(G_{\tilde{\eta}}\right)$ satisfies the Rosati condition defined by the polarization $\lambda \tilde{\eta}$ and by the involution $\star$. (See the theory with endomorphisms in $\$ 7$ below. But beware that we cannot descend $i_{\widetilde{S}}$ to a homomorphism $i: \mathcal{O} \rightarrow \operatorname{End}_{S}(G)$ in general.) When $B=\mathcal{O} \otimes_{\mathbb{Z}} \mathbb{Q}$ is not a division algebra (which is quite often the case), the injective homomorphism $i_{\tilde{\eta}}: \mathcal{O} \rightarrow \operatorname{End}_{\tilde{\eta}}\left(G_{\tilde{\eta}}\right)$ forces $G_{\tilde{\eta}}$ to be isogenous to a non-trivial product of abelian varieties of smaller dimensions, in which case $G_{\eta}$ cannot be absolutely simple.

Example 5.23. In the context of Remark 5.21, suppose $\tilde{k}$ is a quadratic extension of $k$, and suppose $\rho: \operatorname{Gal}(\widetilde{K} / K) \cong \operatorname{Gal}(\widetilde{k} / k) \rightarrow \mathrm{GL}_{2}(\mathbb{Z})$ maps the non-trivial element of $\operatorname{Gal}(\tilde{k} / k)$ to the automorphism $\varsigma$ of $\mathbb{Z}^{2}$ swapping the two factors. Then $\mathcal{O}=\mathbb{Z}[\varsigma]$, and $B=\mathcal{O} \otimes_{\mathbb{Z}} \mathbb{Q}=\mathbb{Q}[\varsigma] \cong \mathbb{Q} \times \mathbb{Q}$ is a product of two fields. Consequently, every $K^{\times}$-valued pairing $\langle\cdot, \cdot\rangle_{\tau, \xi, \xi^{\vee}}$ : $\mathbb{Z}^{2} \times \mathbb{Z}^{2} \rightarrow K^{\times}$satisfying all the conditions in Proposition 5.3 defines an object $(G, \lambda)$ in $\operatorname{DEG}_{\text {pol }}^{\text {tor }}(R)$ such that $G_{\tilde{\eta}}$ is isogenous to the product of two one-dimensional abelian varieties (i.e. elliptic curves) over $\tilde{\eta}$, in which case $G_{\eta}$ cannot be absolutely simple.

Remark 5.24. The proof of Proposition 5.14 avoided the issues in Remarks 5.20 and 5.21 by making crucial use of elements in $\widetilde{K}^{\times}$that are multiplicatively Galois independent as in Lemma 5.10.

§6. Proof of main theorem. Now we are ready to prove our main theorem. Let us first state and prove a finer statement for the case of degenerations over complete discrete valuation rings.

THEOREM 6.1. Let $R, k, K, S, s$, and $\eta$ be as in the beginning of $\S 4$. Given any tori $T_{s}$ and $T_{s}^{\vee}$ with character groups $\underline{X}_{s}$ and $\underline{Y}_{s}$, respectively, and an embedding $\phi_{s}: \underline{Y}_{s} \hookrightarrow \underline{X}_{s}$ inducing an isogeny $\lambda_{T_{s}}=\phi_{s}^{*}: T_{s} \rightarrow T_{s}^{\vee}$ (as in the beginning of $\$ 5)$, there exists an object $(G, \lambda)$ in $\operatorname{DEG}_{\mathrm{pol}}^{\mathrm{tor}}(R)$ (see Definition 4.1) such that $\lambda_{s}: G_{s} \rightarrow G_{s}^{\vee}$ can be identified with $\lambda_{T_{s}}: T_{s} \rightarrow T_{s}^{\vee}$ (via some isomorphisms $G_{s} \cong T_{s}$ and $G_{s}^{\vee} \cong T_{s}^{\vee}$ over $s$ ), and such that $G_{\eta}$ is absolute simple.

Proof. This follows from the combination of Propositions 5.3, 5.5, and 5.14 . 
Thus, the case (a) of Theorem 1.3 over complete discrete valuation rings with residue field $k$ follows from the following.

COROLLARY 6.2. Given any field $k$ and any complete discrete valuation ring $R$ with residue field $k$, any torus over $k$ can be realized as the special fiber of some semi-abelian scheme over $R$ whose generic fiber is an absolutely simple abelian variety (which can be principally polarized) over $K$.

Proof. By Remark 4.3, it suffices to prove the assertion for polarized abelian varieties, and so it suffices to apply Theorem 6.1 to the given torus over $k$, with the isogeny given by the identity morphism from this torus to itself.

It still remains to prove the cases (b) and (c) of Theorem 1.3. We shall deduce them from the case (a) of Theorem 1.3 over complete discrete valuation rings, by slightly modifying the argument in [7, Ch. IV, §4] based on Artin's approximation. Let us retain the setting of Theorem 6.1, but with the more specific choice that $R=k[[t]]$ (respectively the Witt vectors $R=W(k)$ ) in case (b) (respectively in case (c)). Let $I$ denote the maximal ideal of $R$.

Let $R_{0}:=k[t]$ (respectively a number ring with an absolutely unramified prime ideal $\mathfrak{P}$ with residue field $k$, so that $R_{0}$ is embedded in $R$ via $\mathfrak{P}$-adic completion), and let $I_{0}$ denote the ideal $(t)$ (respectively $\mathfrak{P}$ ) of $R_{0}$. Let $R_{1}$ be the Henselization of $R_{0}$ at $I_{0}$, which is canonically a subring of $R$. (See, for example, $[9, I V-4,18.6]$.) Let $I_{1}$ denote the ideal of $R_{1}$ generated by $I_{0}$, so that the maximal ideal $I$ of $R$ is generated by $I_{1}$. For $0 \leqslant j \leqslant 1$, we shall denote the fraction field of $R_{j}$ by $K_{j}$; define $S_{j}:=\operatorname{Spec}\left(R_{j}\right)$ and $\eta_{j}:=\operatorname{Spec}\left(K_{j}\right)$; and, by abuse of notation, still denote by $s$ the closed points $\operatorname{Spec}(k) \rightarrow \operatorname{Spec}\left(R_{j}\right)$ defined by $I_{j}$.

Proposition 6.3. Suppose $R_{2}$ is a subalgebra of $R$ that is of finite type over $R_{1}$. Then, given any integer $m \geqslant 1$, the natural inclusion $R_{1} \hookrightarrow R_{2}$ has some (homomorphic) section $R_{2} \rightarrow R_{1}$ (depending on $m$ ) such that the composition $R_{2} \rightarrow R_{1} \hookrightarrow R$ coincides with the natural inclusion $R_{2} \hookrightarrow R$ after reduction modulo $I^{m}$ in $R$.

Proof. This follows from Artin's approximation, as in [1, Theorem 1.10], because $R_{1}$ is the Henselization of the excellent Dedekind domain $R_{0}$ at the maximal ideal $I_{0}$.

THEOREM 6.4. Given any $(G, \lambda)$ in $\mathrm{DEG}_{\mathrm{pol}}^{\mathrm{tor}}(R)$ as in Theorem 6.1, there exists some $\left(G_{1}, \lambda_{1}\right)$ in $\mathrm{DEG}_{\mathrm{pol}}^{\mathrm{tor}}\left(R_{1}\right)$ (see Remark 4.2) such that $\left(G_{1}, \lambda_{1}\right)$ $\otimes_{R_{1}} k \cong(G, \lambda) \otimes_{R_{1}} k$, and such that the generic fiber $G_{1, \eta_{1}}$ is absolutely simple. Consequently, the special fiber $\lambda_{1, s}: G_{1, s} \rightarrow G_{1, s}^{\vee}$ of $\lambda_{0}$ can also be identified with $\lambda_{T_{s}}: T_{s} \rightarrow T_{s}^{\vee}$ (via some isomorphisms $G_{1, s} \cong T_{s}$ and $G_{1, s}^{\vee} \cong T_{s}^{\vee}$ over $s$ ).

These assertions remain true with $S_{1}=\operatorname{Spec}\left(R_{1}\right)$ replaced with some connected affine étale neighborhood $s \rightarrow U \rightarrow S_{0}=\operatorname{Spec}\left(R_{0}\right)$ of $s$. 
Proof. Since $G \rightarrow S=\operatorname{Spec}(R)$ and $G^{\vee} \rightarrow S$ are both of finite presentation, and since $R$ is the filtering direct limit (union) of its normal subalgebras $R_{2}$ of finite type over $R_{1}$, by [9, IV-3, 8.8.2], we may assume that there exist some such $R_{2}$, some semi-abelian schemes $G_{2} \rightarrow S_{2}:=\operatorname{Spec}\left(R_{2}\right)$ and $G^{\vee} \rightarrow S_{2}$, and some homomorphism $\lambda_{2}: G_{2} \rightarrow G_{2}^{\vee}$ such that $\left(G_{2}, G_{2}^{\vee}, \lambda_{2}\right) \otimes_{R_{2}} R \cong(G$, $\left.G^{\vee}, \lambda\right)$. Since the pullback of $G_{2}$ and $G_{2}^{\vee}$ to $\eta$ are the absolutely simple abelian varieties $G_{\eta}$ and $G_{\eta}^{\vee}$, respectively, by [7, Ch. I, Theorem 2.10] or [14, Ch. 3, Theorem 3.3.1.9]; and by [19, IX, 1.4], [7, Ch. I, Proposition 2.7], or [14, Proposition 3.3.1.5], there exists a non-empty open subset $W$ of $S_{2}$ such that $\left.G_{2}\right|_{W}$ and $\left.G_{2}^{\vee}\right|_{W}$ are abelian schemes whose fibers are all absolutely simple, and such that $\left.\lambda_{2}\right|_{W}$ is a polarization of abelian schemes. Suppose $W$ is the complement of a closed subset of $S_{2}$ defined by some non-zero ideal $J_{2}$ of $R_{2}$. Since $R_{2} \subset R$, and since $R$ is noetherian and $I$-adically separated, there exists an integer $m \geqslant 1$ such that $J_{2}$ is not contained in $I^{m}$. By Proposition 6.3, there exists a section $R_{2} \rightarrow R_{1}$ of $R_{1} \hookrightarrow R_{2}$ such that the composition $R_{2} \rightarrow R_{1} \hookrightarrow R$ coincides with the natural inclusion $R_{2} \hookrightarrow R$ after reduction modulo $I^{m}$ in $R$. Let $\left(G_{1}, G_{1}^{\vee}, \lambda_{1}\right):=\left(G_{2}, G_{2}^{\vee}, \lambda_{2}\right) \otimes_{R_{2}} R_{1}$. Then $\left(G_{1}, G_{1}^{\vee}, \lambda_{1}\right)$ $\otimes_{R_{1}} k \cong\left(G, G^{\vee}, \lambda\right) \otimes_{R} k$ because $m \geqslant 1$. Moreover, $J_{2}$ has non-zero image in $R_{1}$ under the section $R_{2} \rightarrow R_{1}$ above, and therefore the induced morphism $S_{1}=\operatorname{Spec}\left(R_{1}\right) \rightarrow S_{2}=\operatorname{Spec}\left(R_{2}\right)$ maps the generic point $\eta_{1}$ of $S_{1}$ to the above open subset $W$ of $S_{2}$. Hence, $\left(G_{1}, \lambda_{1}\right)$ defines an object in $\operatorname{DEG}_{\mathrm{pol}}^{\text {tor }}\left(R_{1}\right)$, and the generic fiber $G_{1, \eta_{1}}$ is absolutely simple. This proves the first paragraph of the theorem.

Since $G_{1} \rightarrow S_{1}$ and $G_{1}^{\vee} \rightarrow S_{1}$ are both of finite presentation, and since $R_{1}$ is the filtering direct limit of the coordinate rings of all connected affine étale neighborhoods $s \rightarrow U \rightarrow S_{0}$ of $s$, by [9, IV-3, 8.8.2] again, the second paragraph of the theorem follows from the first, as desired.

Now, since an étale neighborhood $s \rightarrow U \rightarrow S_{0}$ of $s$ is necessarily a smooth curve over $k$ (with a $k$-rational point lifting $s$, which can still be denoted $s$ ) in case (b) (respectively an open subset of the spectrum of a number ring in case (c)), the cases (b) and (c) of Theorem 1.3 follow from the following.

COROLLARY 6.5. Given any field $k$ and any complete discrete valuation ring $R$ with residue field $k$, any torus over $k$ can be realized as the special fiber of some semi-abelian scheme over some connected affine étale neighborhood $s \rightarrow$ $U \rightarrow S_{0}$ of $s$ that is generically an absolutely simple abelian variety (which can be principally polarized).

Proof. As explained in the proof of Corollary 6.2, this follows from Theorem 6.4 just as Corollary 6.2 does from Theorem 6.1. (Alternatively, we could have approximated the semi-abelian scheme in Corollary 6.2 directly, using [9, IV-3, 8.8.2] and Proposition 6.3, as in the proof of Theorem 6.4.)

Remark 6.6. By the theory of Néron models (see, in particular, [3, Ch. 7 , $\S 7.4$, Proposition 3]), the semi-abelian schemes in Corollaries 6.2 and 6.5 (where 
the base schemes are Dedekind domains) are exactly the identity components of the Néron models of their respective generic fibers.

§7. Non-trivial endomorphisms. In this section, we investigate the analogue of Question 1.2 which requires additionally that the semi-abelian schemes are equipped with endomorphism structures.

Let $\mathcal{O}$ be an order in a finite-dimensional semisimple algebra $B$ over $\mathbb{Q}$, and let $\star: B \rightarrow B$ be a positive involution (i.e. $\operatorname{Tr}_{B / \mathbb{Q}}\left(x x^{\star}\right)>0$ for all non-zero $x$ in $B$ ), which we assume to stabilize $\mathcal{O}$. All endomorphism algebras of abelian varieties over algebraically closed fields are necessarily of this form, with the involution $\star$ given by the Rosati involution induced by some polarization. (See, for example, [17, $\S 20-21]$, which contains a treatment of Albert's classification of finite-dimensional division algebras over $\mathbb{Q}$ with positive involutions. See also [14, Propositions 1.2.1.13 and 1.2.1.14] for a summary of possibilities when $B$ is simple.)

Let us first expand the review of the theory of degeneration in $\S 4$. Let $R, k$, $K, S, s$, and $\eta$ be as in the beginning of $\S 4$.

Definition 7.1. With the setting as above, the category $\operatorname{DEG}_{\mathrm{PE}, \mathcal{O}}^{\mathrm{tor}}(R)$ has objects consisting of triples $(G, \lambda, i)$ over $S=\operatorname{Spec}(R)$, where $(G, \lambda)$ is an object in $\operatorname{DEG}_{\text {pol }}^{\text {tor }}(R)$, and where $i: \mathcal{O} \rightarrow \operatorname{End}_{S}(G)$ is a homomorphism satisfying the Rosati condition that $\lambda \circ i\left(b^{\star}\right)=(i(b))^{\vee} \circ \lambda$ as homomorphisms from $G$ to $G^{\vee}$, where $(i(b))^{\vee}: G^{\vee} \rightarrow G^{\vee}$ is the unique homomorphism (see [19, IX, 1.4], [7, Ch. I, Proposition 2.7], or [14, Proposition 3.3.1.5]) extending the dual homomorphism $(i(b))_{\eta}^{\vee}: G_{\eta}^{\vee} \rightarrow G_{\eta}^{\vee}$ of $(i(b))_{\eta}: G_{\eta} \rightarrow G_{\eta}$, for all $b \in \mathcal{O}$.

Remark 7.2. As in the case of Definition 4.1 and Remark 4.2, the definition of pairs $(G, \lambda, i)$ as in Definition 4.1 extends verbatim to the case where $S$ is a noetherian normal local scheme.

By the theory of degeneration data (for polarized abelian schemes with endomorphism structures; see [14, §5.1.1; see, in particular, Theorem 5.1.1.4], with all abelian parts in the degenerations suppressed), there is an equivalence of categories

$$
\mathrm{M}_{\mathrm{PE}, \mathcal{O}}^{\mathrm{tor}}(R): \operatorname{DD}_{\mathrm{PE}, \mathcal{O}}^{\mathrm{tor}}(R) \rightarrow \operatorname{DEG}_{\mathrm{PE}, \mathcal{O}}^{\mathrm{tor}}(R):(\underline{X}, \underline{Y}, \phi, \tau) \mapsto(G, \lambda, i)
$$

realizing $(G, \lambda, i)$ (up to isomorphism) as the image of an object in $\operatorname{DD}_{\mathrm{PE}, \mathcal{O}}^{\text {tor }}(R)$ given by the following data.

(1) The tuple $(\underline{X}, \underline{Y}, \phi, \tau)$ is an object in $\mathrm{DD}_{\text {pol }}^{\text {tor }}(R)$, as in $\S 4$.

(2) The étale shaves $\underline{X}$ and $\underline{Y}$ are equipped with the structures of étale sheaves of left $\mathcal{O}$-lattices (i.e. $\mathbb{Z}$-lattices with left $\mathcal{O}$-module structures), and the embedding $\phi: \underline{X} \rightarrow \underline{Y}$ is then $\mathcal{O}$-equivariant.

(3) The bimultiplicative homomorphism $\tau: \underline{Y}_{\eta} \times \underline{X}_{\eta} \rightarrow \mathbb{G}_{\mathrm{m}, \eta}$ is compatible with the actions of $\mathcal{O}$ in the sense that $(b \times 1)^{*} \tau=\left(1 \times b^{\star}\right)^{*} \tau$, for all $b \in \mathcal{O}$. 
We say that $(\underline{X}, \underline{Y}, \phi, \tau)$ is the degeneration data of $(G, \lambda, i)$.

Suppose that we have tori $T_{s}$ and $T_{s}^{\vee}$ over $s=\operatorname{Spec}(k)$ with character groups $\underline{X}_{S}$ and $\underline{Y}_{S}$ that are étale sheaves of left $\mathcal{O}$-lattices, together with an $\mathcal{O}$-equivariant embedding $\phi_{s}: \underline{Y}_{s} \hookrightarrow \underline{X}_{s}$ with finite cokernel, inducing an $\mathcal{O}$-equivariant isogeny $\lambda_{T_{s}}: T_{s} \rightarrow T_{s}^{\vee}$. By Lemma 3.1, the $\mathcal{O}$-equivariant embedding $\phi_{s}$ : $\underline{Y}_{S} \hookrightarrow \underline{X}_{S}$ uniquely lifts to an $\mathcal{O}$-equivariant embedding $\phi: \underline{Y} \hookrightarrow \underline{X}$ over $S$ of étale sheaves of left $\mathcal{O}$-lattices with finite cokernel, inducing an $\mathcal{O}$-equivariant isogeny $\lambda_{T}: T \rightarrow T^{\vee}$ (between isotrivial tori) lifting $\lambda_{T_{s}}$. Let $\widetilde{S}$, etc, be as in $\$ \S 3$ and 5 such that there are left $\mathcal{O}$-lattices $X$ and $Y$, isomorphisms $\xi: X \stackrel{\sim}{\rightarrow} \underline{X}_{\widetilde{\eta}}$ and $\xi^{\vee}: Y \stackrel{\sim}{\rightarrow} \underline{Y}_{\tilde{\eta}}$, and representations $\rho: \operatorname{Gal}(\widetilde{K} / K) \rightarrow \operatorname{GL}_{\mathcal{O}}(X)$ and $\rho^{\vee}: \operatorname{Gal}(\tilde{K} / K) \rightarrow \operatorname{GL}_{\mathcal{O}}(Y)$ defining the descent data for the étale sheaves $\underline{X}_{\eta}$ and $\underline{Y}_{\eta}$ (of left $\mathcal{O}$-lattices). Then we have an $\mathcal{O}$-equivariant embedding

$$
\phi_{\xi, \xi^{\vee}}:=\xi^{-1} \phi_{\widetilde{\eta}} \xi^{\vee}: Y \hookrightarrow X
$$

with finite cokernel (cf. (5.1)), and the following obvious analogue of Lemma 5.2.

LEMMA 7.4. We have

$$
\phi_{\xi, \xi^{\vee}}\left(\rho^{\vee}(\gamma) z\right)=\rho(\gamma)\left(\phi_{\xi, \xi^{\vee}}(z)\right)
$$

for all $\gamma \in \operatorname{Gal}(\tilde{K} / K)$ and $z \in Y$.

Definition 7.5. Given left $\mathcal{O}$-lattices $M$ and $M^{\prime}$, and an abelian group $M^{\prime \prime}$ (such as $\mathbb{Z}$ or $\widetilde{K}^{\times}$), we say that a $(\mathbb{Z}$-)bilinear (or bimultiplicative) pairing $\langle\cdot, \cdot\rangle$ : $M \times M^{\prime} \rightarrow M^{\prime \prime}$ is $\mathcal{O}$-compatible if $\langle b z, w\rangle=\left\langle z, b^{\star} w\right\rangle$ for all $z \in M, w \in M^{\prime}$, and $b \in \mathcal{O}$.

Remark 7.6. By [14, Lemma 1.1.4.5], the $\mathcal{O}$-compatible symmetric bilinear pairings $\langle\cdot, \cdot\rangle: M \times M \rightarrow \mathbb{Z}$ (as in Definition 7.5, with $M=M^{\prime}$ and $M^{\prime \prime}=\mathbb{Z}$ ) are exactly the traces of the Hermitian pairings $(\cdot, \cdot): M \times M \rightarrow \operatorname{Diff}_{\mathcal{O} / \mathbb{Z}}^{-1}$ valued in the inverse different $\operatorname{Diff}_{\mathcal{O} / \mathbb{Z}}^{-1}$ (see [14, Definition 1.1.1.8]).

Then we have the following strengthening of Proposition 5.3.

Proposition 7.7. With the setting as above, with the fixed choices of some $\xi, \xi^{\vee}, \rho$, and $\rho^{\vee}$, the datum of an object $(G, \lambda, i)$ of $\operatorname{DEG}_{\mathrm{PE}, \mathcal{O}}^{\text {tor }}(R)$ (as in Definition 7.1) such that $\lambda_{s}: G_{s} \rightarrow G_{s}^{\vee}$ can be identified with $\lambda_{T_{s}}: T_{s} \rightarrow T_{s}^{\vee}$ (via $\mathcal{O}$-equivariant isomorphisms $G_{s} \cong T_{s}$ and $G_{s}^{\vee} \cong T_{s}^{\vee}$ over $s$ ) is equivalent to the datum of a bimultiplicative pairing

$$
\langle\cdot, \cdot\rangle_{\tau, \xi, \xi^{\vee}}: Y \times X \rightarrow \widetilde{K}^{\times}
$$

satisfying the following conditions. 
(1) (Galois equivariance) $\left\langle\rho^{\vee}(\gamma) z, \rho(\gamma) w\right\rangle_{\tau, \xi, \xi^{\vee}}=\gamma\langle z, w\rangle_{\tau, \xi, \xi^{\vee}}$ for all $z \in$ $Y, w \in X$, and $\gamma \in \operatorname{Gal}(\tilde{K} / K)$.

(2) $\quad$ (Symmetry) $\left\langle z, \phi_{\xi, \xi^{\vee}}(w)\right\rangle_{\tau, \xi, \xi^{\vee}}=\left\langle w, \phi_{\xi, \xi^{\vee}}(z)\right\rangle_{\tau, \xi, \xi^{\vee}}$ for all $z, w \in Y$.

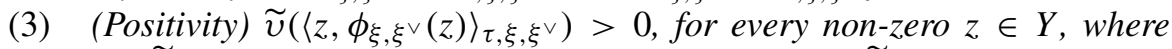
$\widetilde{v}: \widetilde{K}^{\times} \rightarrow \mathbb{Z}$ is any non-trivial discrete valuation of $\widetilde{K}$.

(4) (O-compatibility) $\langle b z, w\rangle_{\tau, \xi, \xi^{\vee}}=\left\langle z, b^{\star} w\right\rangle_{\tau, \xi, \xi^{\vee}}$ for all $z \in Y, w \in X$, and $b \in \mathcal{O}$.

Let $\mathcal{O}_{+}$(respectively $\mathcal{O}_{-}$) denote the $\mathbb{Z}$-submodule of $\mathcal{O}$ consisting of $b \in \mathcal{O}$ such that $b^{\star}=b$ (respectively $b^{\star}=-b$ ). Note that $\mathcal{O}_{+} \cap \mathcal{O}_{-}=0$ and $2 \mathcal{O} \subset \mathcal{O}_{+}+$ $\mathcal{O}-\subset \mathcal{O}$, because $2 b=\left(b+b^{\star}\right)+\left(b-b^{\star}\right)$ for all $b \in \mathcal{O}$. Let $B_{+}$(respectively $B_{-}$) denote the $\mathbb{Q}$-subspace of $B=\mathcal{O} \otimes_{\mathbb{Z}} \mathbb{Q}$ spanned by $\mathcal{O}_{+}$(respectively $\mathcal{O}_{-}$). Then $\mathcal{O}_{+}+\mathcal{O}-\subset \mathcal{O}$ induces $B_{+} \oplus B_{-} \cong B$.

We have the following rather elaborate analogue of Proposition 5.5.

Proposition 7.9. In the setting of Proposition 7.7, suppose that $B=\mathcal{O} \otimes_{\mathbb{Z}}$ $\mathbb{Q}$ is a division algebra. Suppose that the pairing $\langle\cdot, \cdot\rangle_{\tau, \xi, \xi \vee}$ corresponding to $(G, \lambda)$ satisfies the additional condition that

$$
\langle z, w\rangle_{\tau, \xi, \xi \vee} \neq 1
$$

for all non-zero $z \in Y$ and $w \in X$, except when $\phi_{\xi, \xi^{\vee}}(z)=c w$ for some nonzero $c \in B_{-}$. Then $G_{\eta}$ and hence $G_{\eta}^{\vee}$ must be absolutely simple, except when $B$ belongs to one of the following two special cases (see Remark 7.12 below).

(A) The center $F$ of the algebra $B$ is a totally real field, and $B \otimes_{F, \sigma} \mathbb{R}$ is isomorphic to the Hamilton quaternion algebra $\mathbb{H}$ over $\mathbb{R}$ for every field homomorphism $\sigma: F \rightarrow \mathbb{R}$, with the positive involution $\star$ of $B$ induced by the canonical positive involution $x \mapsto \operatorname{Tr}_{\mathbb{H} / \mathbb{R}}(x)-x$ on $\mathbb{H}$. (This is exactly the third case in [14, Proposition 1.2.1.13].) Also, $Y \otimes_{\mathbb{Z}} \mathbb{Q} \cong B$ as $B$-modules.

(B) The center $F$ of the algebra $B$ is a CM field (i.e. is a totally imaginary extension of a totally real field $F_{+}$), and $B=F$. (This is a very special case of the fourth case in [14, Proposition 1.2.1.13].) Also, $Y \otimes_{\mathbb{Z}} \mathbb{Q} \cong B$ as B-modules.

Remark 7.11. The assumption that $B=\mathcal{O} \otimes_{\mathbb{Z}} \mathbb{Q}$ is a division algebra is natural for our purpose, because the existence of any non-trivial idempotent in $B$ will force $G_{\eta}$ to be isogenous to a non-trivial product of abelian varieties of smaller dimensions.

Remark 7.12. The two exceptional cases (A) and (B) in Proposition 7.9 (and therefore also in Theorems 7.20, 7.22, and 7.24 below) are unavoidable for the following reasons. Suppose $\langle\cdot, \cdot\rangle_{\tau, \xi, \xi \vee}$ is any pairing, with associated $G$ over $S$, as in Proposition 7.7. For simplicity, let us identify $Y$ with its image $\phi_{\xi, \xi^{\vee}}(Y)$ in $X$, and suppress $\phi_{\xi, \xi \vee}$ from the notation. In the case (A), there exist $e, f \in \mathcal{O}_{-}$ such that $B=B^{\prime} \oplus f B^{\prime}$ as modules of the (commutative) CM field $B^{\prime}:=F+F e$, 
by letting $B^{\prime}$ act on $B$ by right multiplication. Since $f^{\star}=-f$, for any $z \in Y \subset$ $X$, we have

$$
\begin{aligned}
\langle a z, 4 f b z\rangle_{\tau, \xi, \xi^{\vee}} & =\langle a z, 2 f b z\rangle_{\tau, \xi, \xi^{\vee}} \cdot\langle-2 f a z, b z\rangle_{\tau, \xi, \xi^{\vee}} \\
& =\left\langle z, 2\left(a^{\star} f b-b^{\star} f a\right) z\right\rangle_{\tau, \xi, \xi^{\vee}} \\
& =\left\langle z,\left(a^{\star} f b-b^{\star} f a\right) z\right\rangle_{\tau, \xi, \xi^{\vee}} \cdot\left\langle\left(b^{\star} f a-a^{\star} f b\right) z, z\right\rangle_{\tau, \xi, \xi^{\vee}} \\
& =1,
\end{aligned}
$$

for all $a, b \in \mathcal{O}^{\prime}:=\mathcal{O} \cap B^{\prime}$. Similarly, in the case (B), there exists $e \in \mathcal{O}_{-}$ such that $B=F=B^{\prime} \oplus B^{\prime} e$ as modules of the (commutative) totally real field $B^{\prime}:=F_{+}$. Since $e^{\star}=-e$, and since $B=F$ is commutative, for any $z \in Y \subset X$, we have

$$
\langle a z, 2 b e z\rangle_{\tau, \xi, \xi^{\vee}}=\langle a b z, e z\rangle_{\tau, \xi, \xi^{\vee}} \cdot\langle-e z, a b z\rangle_{\tau, \xi, \xi^{\vee}}=1,
$$

for all $a, b \in \mathcal{O}^{\prime}:=\mathcal{O}_{+}=\mathcal{O} \cap B^{\prime}$. Hence, in both cases, up to replacing $Y$ and $X$ with some sublattices of finite indices, the pairing $\langle\cdot, \cdot\rangle_{\tau, \xi, \xi^{\vee}}$ decomposes into an orthogonal direct sum of two pairings that are symmetric, positive, and compatible with the order $\mathcal{O}^{\prime}$ in $B^{\prime}$ (but not necessarily Galois equivariant) as in Proposition 7.7. Therefore, the base change $G_{\bar{\eta}}$ of $G_{\eta}$ to any geometric point $\bar{\eta} \rightarrow S$ above $\eta \rightarrow S$ is isogenous to a product of two abelian varieties over $\bar{\eta}$ with endomorphisms by $\mathcal{O}^{\prime}$, both of which are simple by applying Proposition 7.9 with $\mathcal{O}$ replaced with $\mathcal{O}^{\prime}$. (Moreover, these two simple abelian varieties are isogenous to each other by the same argument as in the second paragraph of the proof of Proposition 7.9 below.) In particular, $G_{\eta}$ can never be absolutely simple in these two exceptional cases.

Proof of Proposition 7.9. Without loss of generality, we may and we shall assume that $Y \neq 0$, so that $G_{\eta}$ is a non-trivial abelian variety over $\eta$.

The condition (7.10) implies the weaker condition that, for any given nonzero $z \in Y$ and $w \in X$, there exists some $b \in \mathcal{O}$ such that $\langle v, b w\rangle_{\tau, \xi, \xi \vee} \neq 1$. By the same argument as in the proof of Proposition 5.5, this implies that $G_{\eta}$ and hence $G_{\eta}^{\vee}$ are absolutely simple in the category of abelian varieties with endomorphisms by $\mathcal{O}$, in the sense that their base changes $G_{\bar{\eta}}$ and $G_{\bar{\eta}}^{\vee}$ to any geometric point $\bar{\eta} \rightarrow S$ above $\eta \rightarrow S$ are not isogenous to non-trivial products of abelian varieties of smaller dimensions with endomorphisms by $\mathcal{O}$. Moreover, if $G_{\bar{\eta}}$ is isogenous to a product $A_{1} \times \bar{\eta} A_{2} \times \bar{\eta} \cdots \times \times_{\bar{\eta}} A_{r}$ of simple abelian subvarieties over $\bar{\eta}$, then the actions of elements of $\mathcal{O}$ on $G_{\bar{\eta}}$ induce quasi-isogenies between all possible pairs $A_{i}$ and $A_{j}$, with $1 \leqslant i, j \leqslant r$, because otherwise $G_{\bar{\eta}}$ cannot be absolutely simple in the category of abelian varieties with endomorphisms by $\mathcal{O}$. Hence, we may assume that $G_{\bar{\eta}}$ is isogenous to $A^{r}$ for some (non-trivial) simple abelian variety $A$ over $\bar{\eta}$, for some integer $r \geqslant 1$. Note that $\operatorname{dim}(A) \leqslant \frac{1}{2} \operatorname{dim}\left(G_{\bar{\eta}}\right)$ when $r \geqslant 2$.

For simplicity, let us identify $Y$ with its image $\phi_{\xi, \xi \vee}(Y)$ in $X$, and suppress $\phi_{\xi, \xi \vee}$ from the notation. Suppose $e_{1}, e_{2}, \ldots, e_{n}$ are elements of $Y$ such that the assignment $B^{n} \rightarrow Y \otimes_{\mathbb{Z}} \mathbb{Q}:\left(a_{i}\right)_{1 \leqslant i \leqslant n} \mapsto \sum_{1 \leqslant i \leqslant n} a_{i} e_{i}$ induces an isomorphism 
of left $B$-modules (which is possible because $B$ is a division algebra, whose simple modules are all isomorphic to $B$ itself; see [14, Lemma 1.1.2.4]).

Suppose $n \geqslant 2$. Then we have $\left\langle e_{1}, b e_{j}\right\rangle_{\tau, \xi, \xi^{\vee}} \neq 1$ for all $j>1$ and all $b \in \mathcal{O}$. Note that the dimension of the $\mathbb{Q}$-span of $e_{1}$ and all the $b e_{j}$ for all $j>1$ and all $b \in \mathcal{O}$ is greater than $(n-1) \operatorname{dim}_{\mathbb{Q}}(B)=((n-1) / n) \operatorname{dim}\left(G_{\bar{\eta}}\right) \geqslant \frac{1}{2} \operatorname{dim}\left(G_{\bar{\eta}}\right)$. This shows that $\operatorname{dim}(A)>\frac{1}{2} \operatorname{dim}\left(G_{\bar{\eta}}\right)$, so that $r=1$. That is, $G_{\bar{\eta}}$ is simple whenever $n \geqslant 2$.

Suppose $n=1$, so that $\operatorname{dim}\left(G_{\bar{\eta}}\right)=\operatorname{dim}_{\mathbb{Q}}(B)$. Let $F$ denote the center of $B$. We have four cases of the division algebra $B$ with the positive involution $\star$, as in $[17, \S 21]$ or [14, Proposition 1.2.1.13].

(I) The center $F$ is a totally real field, and $B=F$, with the trivial involution $\star$. In this case, we have $B_{-}=0$, and $G_{\bar{\eta}}$ is simple by Proposition 5.5 , because the condition (7.10) here is identical to the corresponding condition (5.6) there.

(II) The center $F$ is a totally real field, and $B \otimes_{F, \sigma} \mathbb{R}$ is isomorphic to $\mathrm{M}_{2}(\mathbb{R})$ over $\mathbb{R}$ for every field homomorphism $\sigma: F \rightarrow \mathbb{R}$, with the positive involution $\star$ of $B$ induced by some conjugation of the canonical positive involution $x \mapsto{ }^{t} x$ of $\mathrm{M}_{2}(\mathbb{R})$. In this case, since $\left\langle e_{1}, b e_{1}\right\rangle_{\tau, \xi, \xi^{\vee}} \neq 1$ for all $b \in \mathcal{O}_{+}$, and since $\operatorname{dim}_{\mathbb{Q}}\left(B_{+}\right)=\frac{3}{4} \operatorname{dim}_{\mathbb{Q}}(B)>\frac{1}{2} \operatorname{dim}_{\mathbb{Q}}(B)=\frac{1}{2} \operatorname{dim}\left(G_{\bar{\eta}}\right)$, we can conclude as before that $r=1$, so that $G_{\bar{\eta}}$ is simple.

(III) The center $F$ is a totally real field, and $B \otimes_{F, \sigma} \mathbb{R}$ is isomorphic to the Hamilton quaternion algebra $\mathbb{H}$ over $\mathbb{R}$ for every field homomorphism $\sigma$ : $F \rightarrow \mathbb{R}$. This is exactly the exceptional case (A) in the proposition.

(IV) The center $F$ is a totally imaginary extension of a totally real field $F_{+}$, with the restriction of the involution $\star$ to $F$ given by the complex conjugation over $F_{+}$. (We shall ignore the additional conditions satisfied by $B$.) In this case, since $\left\langle e_{1}, b e_{1}\right\rangle_{\tau, \xi, \xi^{\vee}} \neq 1$ for all $b \in \mathcal{O}_{+}$, and since $\operatorname{dim}_{\mathbb{Q}}\left(B_{+}\right)=\frac{1}{2} \operatorname{dim}_{\mathbb{Q}}(B)$, we have $r \leqslant 2$. If $r=1$, then $G_{\bar{\eta}}$ is simple, as desired.

Otherwise, we necessarily have $r=2$. Let $D:=\operatorname{End}_{\bar{\eta}}(A) \otimes_{\mathbb{Z}} \mathbb{Q}$, which is a division algebra with a positive involution, because $A$ is a simple abelian variety. (See, for example, $[\mathbf{1 7}, \S \S 20-21]$.) Then we have an injective homomorphism $B \hookrightarrow \mathrm{M}_{2}(D)$ of algebras over $\mathbb{Q}$, which cannot be an isomorphism because $B$ is a division algebra but $\mathrm{M}_{2}(D)$ is not. Since the action of the order $\operatorname{End}_{\bar{\eta}}(A)$ in $D$ induces an action of the same order on the character group of the torus degeneration of $A$, we have an action of $D$ on $B$ stabilizing the $\mathbb{Q}$-subspaces $B_{+}$and $B_{-}$ of the same dimension, so that $B \cong B_{+} \oplus B_{-}$and $B_{+} \cong D^{r^{\prime}} \cong B_{-}$ as $D$-modules, for some integer $r^{\prime} \geqslant 1$. Then we have $2 r^{\prime} \operatorname{dim}_{\mathbb{Q}}(D) \leqslant$ $\operatorname{dim}_{\mathbb{Q}}(B)<4 \operatorname{dim}_{\mathbb{Q}}(D)$, forcing $r^{\prime}=1$.

Let $E$ denote the center of $D$, which is a field and is also the center of $\mathrm{M}_{2}(D)$. Then $E_{0}:=B \cap E$ is a subfield of the center $F$ of $B$, because $E$ commutes with all elements of $B$. If $E_{0}=E$, then $E$ is a subfield of $F$. Otherwise, the $E$-span $B^{\prime}$ of the image of $B$ in $\mathrm{M}_{2}(D)$ form an $E$ - 
subalgebra of $\mathrm{M}_{2}(D)$ such that $\operatorname{dim}_{\mathbb{Q}}\left(B^{\prime}\right) \geqslant 2 \operatorname{dim}_{\mathbb{Q}}(B)=4 \operatorname{dim}_{\mathbb{Q}}(D)$, which must be the whole of $\mathrm{M}_{2}(D)$. Therefore, $B \otimes_{E_{0}} E \cong \mathrm{M}_{2}(D)$ is simple, which implies that $F=E_{0}$. In particular, we have either $E \subset F$ or $F \subset E$.

Since $E$ is the center of an endomorphism algebra of a simple abelian variety, it is either a totally real field or a CM field. If $E$ is a CM field, then there exists some non-zero $c \in E_{0}=E \cap F$ such that $c^{\star}=-c$, so that $c B_{+}=B_{-}$, contradicting the assumption that $D$ stabilizes $B_{+}$. Hence, $E$ is necessarily a totally real subfield, in which case $[D: E]$ is either 4 or 1 , and $E \subset F$ (because $F \subset E$ cannot happen, since $F$ is a $C M$ field).

Let $C$ denote the centralizer of $B$ in $\mathrm{M}_{2}(D)$. By [10, Ch. 5, $\S 11$, Theorem 19], we have $[B: E][C: E]=\left[\mathrm{M}_{2}(D): E\right]=4[D: E]$.

If $[D: E]=4$, then $[F: E]^{2} \mid[B: E][C: E]=16$, and so $[F:$ $E]$ is either 4 or 2 , and $[B: F]$ is either 1 or 4 . If $[B: F]=1$, then the torus degeneration of $A$ is of dimension $\frac{1}{2} \operatorname{dim}_{\mathbb{Q}}(B)=\frac{1}{2} \operatorname{dim}_{\mathbb{Q}}(F) \leqslant$ $2 \operatorname{dim}_{\mathbb{Q}}(E)$, which cannot admit an action of the quaternion algebra $D$ over $E$. If $[B: F]=4$, then $[F: E]=2$ and $C=F$, forcing $F_{+}=E$. Consider any $f \in F$ such that $f^{\star}=-f$, so that $F=E[f]=E+E f$. Then $B_{-}=B_{+} \cdot f$ and $B_{+}=B_{-} \cdot f$, and the left actions of $D$ and $F$ on $B$ commute with each other because the left action of $f$ on $B$ can be equivalently given by the right multiplication by $f$, and because $f^{2} \in$ $F_{+}=E$ commutes with $D$. Then $D \otimes_{E} F$ can be canonically identified with an $E$-subalgebra of $\mathrm{M}_{2}(D)$, and the centralizers of $E \subset F \subset \mathrm{M}_{2}(E)$ are $\mathrm{M}_{2}(D) \supset D \otimes_{E} F \supset D$, respectively. Consequently, $B=D \otimes_{E} F$ as $E$-subalgebras of $\mathrm{M}_{2}(D)$, because the centralizer of $C=F$ is $B$, by $[10$, Ch. 5, §11, Theorem 19] again. Since the positive involution $\star$ of $B$ restricts to a positive involution on $D$, and since this restriction cannot be trivial because $D$ is a quaternion algebra over $E$ (and because of Albert's classification), there exists some non-zero $d \in D \cap B_{-}$. But then $d^{-1}$ exists in $D$, and the left multiplication by $d^{-1} \in D$ maps $d \in B_{-}$to the non-zero $1 \in B_{+}$, contradicting the assumption that $D$ stabilizes $B_{-}$.

Finally, if $[D: E]=1$, then $[F: E]^{2} \mid[B: E][C: E]=4$, and so $[F: E]=2$ and $B=F=C$. This is exactly the exceptional case (B) in the proposition.

Thus, we have shown that $G_{\eta}$ and, hence, $G_{\eta}^{\vee}$ are absolutely simple except in the two cases (A) and (B) in the proposition, as desired.

Hence, the key point is to establish the following analogue of Proposition 5.14.

PROPOSITION 7.13. Suppose that $B=\mathcal{O} \otimes_{\mathbb{Z}} \mathbb{Q}$ is a division algebra. Then there exists a bimultiplicative pairing

$$
\langle\cdot, \cdot\rangle_{\tau, \xi, \xi^{\vee}}: Y \times X \rightarrow \widetilde{K}^{\times}
$$

as in (7.8) satisfying all the conditions in Propositions 7.7 and 7.9. 
Proof. The idea is similar to that in the proof of Proposition 5.14, but the implementation here is, perhaps unavoidably, more tedious. Let

$$
\langle\cdot, \cdot\rangle_{0}: X \times X \rightarrow \mathbb{Z}
$$

be any positive definite $\mathcal{O}$-compatible (symmetric) bilinear pairing (which exists by the same argument as in the proof of, for example, [13, Lemma 2.5]).

Suppose $e_{1}, e_{2}, \ldots, e_{n}$ are elements of $X \otimes_{\mathbb{Z}} \mathbb{Q}$ such that the assignment $B^{n} \rightarrow X \otimes_{\mathbb{Z}} \mathbb{Q}:\left(a_{i}\right)_{1 \leqslant i \leqslant n} \mapsto \sum_{1 \leqslant i \leqslant n} a_{i} e_{i}$ induces an isomorphism of left $B$-modules (which, again, is possible because $B$ is a division algebra, whose simple modules are all isomorphic to $B$ itself; see [14, Lemma 1.1.2.4]), and such that $X$ is contained in the left $\mathcal{O}$-lattice $Z:=\sum_{1 \leqslant i \leqslant n}\left(\mathcal{O} e_{i}\right)$, which is the image of $\mathcal{O}^{n}$ under the last isomorphism. Then there exists a sufficiently divisible integer $r \in \mathbb{Z}_{\geqslant 1}$ such that $r\left\langle e_{i}, b e_{j}\right\rangle_{0} \in \mathbb{Z}$ for all $b \in \mathcal{O}$ and $1 \leqslant i, j \leqslant n$; namely, $r\langle\cdot, \cdot\rangle_{0}$ induces a positive definite $\mathcal{O}$-compatible (symmetric) bilinear pairing $Z \times Z \rightarrow \mathbb{Z}$.

Let $\varepsilon_{1}, \varepsilon_{2}, \ldots, \varepsilon_{h}$ be a $\mathbb{Z}$-basis of $\mathcal{O}_{+}$, and let $\varepsilon_{h+1}, \varepsilon_{h+2}, \ldots, \varepsilon_{m}$ be a $\mathbb{Z}$-basis of $\mathcal{O}_{-}$. Let $\varpi$ be any element of $K^{\times}$of positive valuation. Let $\left\{u_{i j l}\right\}_{1 \leqslant i \leqslant j \leqslant m ; 1 \leqslant l \leqslant m_{i j}^{\prime}}$, where $m_{i j}^{\prime}=h$ when $i=j$ and $m_{i j}^{\prime}=n$ when $i<j$, be elements in $\widetilde{R}^{\times}$that are multiplicatively independent as in Lemma 5.10. Then we define a symmetric bimultiplicative pairing

$$
\langle\cdot, \cdot\rangle_{1}: Z \times Z \rightarrow \widetilde{K}^{\times}
$$

satisfying the compatibility

$$
\langle b z, w\rangle_{1}=\left\langle z, b^{\star} w\right\rangle_{1}
$$

for all $b \in \mathcal{O}_{+} \oplus \mathcal{O}_{-}$and $z, w \in Z$ by setting:

(1) $\left\langle e_{i}, \varepsilon_{l} e_{i}\right\rangle_{1}:=u_{i j l} \cdot \varpi^{r\left\langle e_{i}, \varepsilon_{l} e_{i}\right\rangle_{0}}$ for all $1 \leqslant i \leqslant n$ and $1 \leqslant l \leqslant h$ (in which case $\varepsilon_{l}^{\star}=\varepsilon_{l}$ is compatible with $\left.\left\langle e_{i}, \varepsilon_{l}^{\star} e_{i}\right\rangle_{0}=\left\langle\varepsilon_{l}^{\star} e_{i}, e_{i}\right\rangle_{0}=\left\langle e_{i}, \varepsilon_{l} e_{i}\right\rangle_{0}\right)$;

(2) $\left\langle e_{i}, \varepsilon_{l} e_{i}\right\rangle_{1}:=1$ for all $1 \leqslant i \leqslant n$ and $h<l \leqslant m$ (in which case $\varepsilon_{l}^{\star}=-\varepsilon_{l}$ forces $\left.\left\langle e_{i}, \varepsilon_{l}^{\star} e_{i}\right\rangle_{0}=\left\langle\varepsilon_{l}^{\star} e_{i}, e_{i}\right\rangle_{0}=\left\langle e_{i}, \varepsilon_{l} e_{i}\right\rangle_{0}=0\right)$; and

$$
\left\langle e_{i}, \varepsilon_{l} e_{j}\right\rangle_{1}:=u_{i j l} \cdot \varpi^{r\left\langle e_{i}, \varepsilon_{l} e_{j}\right\rangle_{0}} \text { for all } 1 \leqslant i<j \leqslant n \text { and } 1 \leqslant l \leqslant m \text {, }
$$

and by extending the values of the pairing to the whole domain $Z \times Z$ by symmetry, bimultiplicativity, and the rule that $\left\langle a e_{i}, b e_{j}\right\rangle_{1}:=\left\langle e_{i}, a^{\star} b e_{j}\right\rangle_{1}$ for all $a, b \in \mathcal{O}_{+} \oplus \mathcal{O}_{-}$and $1 \leqslant i \leqslant j \leqslant n$. Note that this is well defined because $B e_{i} \cong B$ as left $B$-modules, for all $1 \leqslant i \leqslant n$. Also, note that $\widetilde{v} \circ\langle\cdot, \cdot\rangle_{1}$ is a positive multiple of $\langle\cdot, \cdot\rangle_{0}$ for every non-trivial discrete valuation $\widetilde{v}: \widetilde{K}^{\times} \rightarrow \mathbb{Z}$.

$\mathrm{Next}$, we define an $\mathcal{O}$-compatible symmetric bilinear pairing

$$
\langle\cdot, \cdot\rangle_{2}: X \times X \rightarrow \widetilde{K}^{\times}
$$

satisfying the Galois equivariance

$$
\langle\rho(\gamma) z, \rho(\gamma) w\rangle_{2}=\gamma\langle z, w\rangle_{2}
$$


for all $\gamma \in \operatorname{Gal}(\widetilde{K} / K)$ and $z, w \in X$ (cf. (5.15)) by setting

$$
\langle z, w\rangle_{2}:=\prod_{\gamma \in \operatorname{Gal}(\widetilde{K} / K)} \gamma^{-1}\langle\rho(\gamma) z, \rho(\gamma) w\rangle_{1}
$$

for all $z, w \in X$. Note that $\langle\rho(\gamma) z, \rho(\gamma) w\rangle_{1}$ are defined for all $z, w \in X$ because $X$ is contained in $Z$, and that $\widetilde{v} \circ\langle\cdot, \cdot\rangle_{2}$ is still positive definite for every non-trivial discrete valuation $\widetilde{v}: \widetilde{K}^{\times} \rightarrow \mathbb{Z}$.

Finally, we define the desired bimultiplicative pairing

$$
\langle\cdot, \cdot\rangle_{\tau, \xi, \xi^{\vee}}: Y \times X \rightarrow \widetilde{K}^{\times}
$$

by setting

$$
\langle z, w\rangle_{\tau, \xi, \xi^{\vee}}:=\left\langle\phi_{\xi, \xi^{\vee}}(z), 2 w\right\rangle_{2}
$$

for all $z \in Y$ and $w \in X$, where $\phi_{\xi, \xi \vee}$ is as in (7.3) (cf. (5.17); but note the coefficient 2 of $w$ ). Then $\langle\cdot, \cdot\rangle_{\tau, \xi, \xi \vee}$ satisfies the first three conditions (1), (2), and (3) in Proposition 7.7 by the symmetry and positive definiteness of $\langle\cdot, \cdot\rangle_{0}$; by the definitions of the pairings $\langle\cdot, \cdot\rangle_{1}$ and $\langle\cdot, \cdot\rangle_{2}$; by the choices of $\varpi$ (of positive valuation in $\widetilde{K}^{\times}$) and $\left\{u_{i j l}\right\}_{1 \leqslant i \leqslant j \leqslant n ; 1 \leqslant l \leqslant m_{i j}^{\prime}}$ (of zero valuation in $\widetilde{K}^{\times}$); and by Lemma 7.4 and the relations (7.15) and (7.17). As for the remaining condition (4) in Proposition 7.7, it suffices to note that, since $2 \mathcal{O} \subset \mathcal{O}_{+} \oplus \mathcal{O}_{-}$, the compatibility (7.14) implies that $\langle b z,(2 w)\rangle_{2}=\langle(2 b) z, w\rangle_{2}=\left\langle z,\left(2 b^{\star}\right) w\right\rangle_{2}=$ $\left\langle z, b^{\star}(2 w)\right\rangle_{2}$ for all $b \in \mathcal{O}$ and $z, w \in Z$.

It remains to show that $\langle z, w\rangle_{\tau, \xi, \xi^{\vee}} \neq 1$ (as in (7.10)) for all non-zero $z \in$ $Y$ and $w \in X$, except when $\phi_{\xi, \xi^{\vee}}(z)=c w$ for some non-zero $c \in B_{-}$. Since $\phi_{\xi, \xi \vee}$ is an embedding, by the defining relation (7.17), it suffices to show that $\langle z, 2 w\rangle_{2} \neq 1$ for all non-zero $z, w \in Z$, except when $z=c w$ for some non-zero $c \in B_{-}$. By the choice of $\left\{u_{i j l}\right\}_{1 \leqslant i \leqslant j \leqslant n ; 1 \leqslant l \leqslant m_{i j}^{\prime}}$, the terms in the product (7.16) indexed by different elements $\gamma$ have multiplicatively independent values (up to powers of $\varpi)$, and so it suffices to show that $\langle z, 2 w\rangle_{1} \neq 1$ for all non-zero $z, w \in Z$, except when $z=c w$ for some non-zero $c \in B_{-}$. Suppose that there are some $z=\sum_{1 \leqslant i \leqslant n}\left(a_{i} e_{i}\right)$ and $w=\sum_{1 \leqslant i \leqslant n}\left(b_{i} e_{i}\right)$ in $Z$, where $\left(a_{i}\right)_{1 \leqslant i \leqslant n}$ and $\left(b_{i}\right)_{1 \leqslant i \leqslant n}$ are non-zero $n$-tuples of elements in $\mathcal{O}$, such that $\langle z, 2 w\rangle_{1}=1$. Then we have

$$
\left(\prod_{1 \leqslant i \leqslant n}\left\langle e_{i}, 2 a_{i}^{\star} b_{i} e_{i}\right\rangle_{1}\right) \cdot\left(\prod_{1 \leqslant i<j \leqslant n}\left\langle e_{i}, 2\left(a_{i}^{\star} b_{j}+b_{i}^{\star} a_{j}\right) e_{j}\right\rangle_{1}\right)=1
$$

in $\widetilde{K}^{\times}$(using again the relation $2 \mathcal{O} \subset \mathcal{O}_{+} \oplus \mathcal{O}_{-}$and the compatibility (7.14)). By pulling out all powers of $\varpi$, and by the choice of $\left\{u_{i j l}\right\}_{1 \leqslant i \leqslant j \leqslant n ; 1 \leqslant l \leqslant m_{i j}^{\prime}}$ again, the identity (7.18) is possible only when

$$
a_{i}^{\star} b_{j}+b_{i}^{\star} a_{j}=0
$$

in $B$, for all $1 \leqslant i \leqslant j \leqslant n$. Let $i_{0}$ (respectively $j_{0}$ ) be the smallest index $i$ (respectively $j$ ) such that $a_{i} \neq 0$ (respectively $b_{j} \neq 0$ ), which exists by 
assumption. If $i_{0} \neq j_{0}$, then $a_{i_{0}}^{\star} b_{j_{0}}+b_{i_{0}}^{\star} a_{j_{0}} \neq 0$, which contradicts the condition (7.19). If $i_{0}=j_{0}$, then the condition (7.19) implies that

$$
c:=a_{i_{0}} b_{i_{0}}^{-1}
$$

is a non-zero element of $B_{-}$, because

$c^{\star}=\left(b_{i_{0}}^{\star}\right)^{-1} a_{i_{0}}^{\star}=\left(b_{i_{0}}^{\star}\right)^{-1}\left(a_{i_{0}}^{\star} b_{i_{0}}\right) b_{i_{0}}^{-1}=\left(b_{i_{0}}^{\star}\right)^{-1}\left(-b_{i_{0}}^{\star} a_{i_{0}}\right) b_{i_{0}}^{-1}=-a_{i_{0}} b_{i_{0}}^{-1}=-c$.

Moreover, for every $j \geqslant i_{0}$, the condition (7.19) implies that

$$
a_{j}=\left(b_{i_{0}}^{\star}\right)^{-1}\left(b_{i_{0}}^{\star} a_{j}\right)=\left(b_{i_{0}}^{\star}\right)^{-1}\left(-a_{i_{0}}^{\star} b_{j}\right)=-c^{\star} b_{j}=c b_{j} .
$$

Thus, $z=c w$ for some non-zero $c \in B_{-}$, as desired.

Based on the combination of Propositions 7.7, 7.9, and 7.13, the same arguments as before give the following analogues of Theorem 6.1, Corollary 6.2, Theorem 6.4, and Corollary 6.5.

THEOREM 7.20. Let $R, k, K, S, s$, and $\eta$ be as in the beginning of $\S 4$. Let $\mathcal{O}$ be as in the beginning of this $\S 7$, but assume moreover that $B=\mathcal{O} \otimes_{\mathbb{Z}} \mathbb{Q}$ is a division algebra. Given any tori $T_{s}$ and $T_{s}^{\vee}$ over $s$ with character groups $\underline{X}_{s}$ and $\underline{Y}_{s}$, respectively, that are étale sheaves of left $\mathcal{O}$-lattices, and an $\mathcal{O}$ equivariant embedding $\phi_{s}: \underline{Y}_{s} \hookrightarrow \underline{X}_{s}$ with finite cokernel, inducing an $\mathcal{O}$ equivariant isogeny $\lambda_{T_{s}}=\phi_{s}^{*}: T_{s} \rightarrow T_{s}^{\vee}$, there exists an object $(G, \lambda, i)$ in $\mathrm{DEG}_{\mathrm{PE}, \mathcal{O}}^{\mathrm{tor}}(R)$ (see Definition 7.1) such that $\lambda_{s}: G_{s} \rightarrow G_{s}^{\vee}$ can be identified with $\lambda_{T_{s}}: T_{s} \rightarrow T_{s}^{\vee}$ (via some $\mathcal{O}$-equivariant isomorphisms $G_{s} \cong T_{s}$ and $G_{s}^{\vee} \cong T_{s}^{\vee}$ over $s)$, and such that $G_{\eta}$ is absolutely simple except in the two cases (A) and (B) in Proposition 7.9.

COROLlary 7.21. Let $\mathcal{O}$ be as in Theorem 7.20. Given any field $k$ and any complete discrete valuation ring $R$ with residue field $k$, any torus with endomorphisms by $\mathcal{O}$ over $k$ can be realized as the special fiber of some semi-abelian scheme over $R$ whose generic fiber is an abelian variety with endomorphisms by $\mathcal{O}$ over $K$ (which can be principally polarized) that is absolutely simple except in the two cases $(A)$ and $(B)$ in Proposition 7.9.

THEOREM 7.22. Let $\mathcal{O}$ be as in Theorem 7.20. Given any $(G, \lambda, i)$ in $\operatorname{DEG}_{\mathrm{PE}, \mathcal{O}}^{\mathrm{tor}}(R)$ as in Theorem 7.20, there exists some $\left(G_{1}, \lambda_{1}, i_{1}\right)$ in $\operatorname{DEG}_{\mathrm{PE}, \mathcal{O}}^{\mathrm{tor}}\left(R_{1}\right)$ (see Remark 7.2) such that $\left(G_{1}, \lambda_{1}, i_{1}\right) \otimes_{R_{1}} k \cong(G, \lambda, i) \otimes_{R_{1}} k$, and such that the generic fiber $G_{1, \eta_{1}}$ is absolutely simple except in the two cases (A) and (B) in Proposition 7.9. Consequently, the special fiber $\lambda_{1, s}: G_{1, s} \rightarrow G_{1, s}^{\vee}$ of $\lambda_{1}$ can also be identified with $\lambda_{T_{s}}: T_{s} \rightarrow T_{s}^{\vee}$ (via some $\mathcal{O}$-equivariant isomorphisms $G_{1, s} \cong T_{s}$ and $G_{1, s}^{\vee} \cong T_{s}^{\vee}$ over $s$ ).

These assertions remain true with $S_{1}=\operatorname{Spec}\left(R_{1}\right)$ replaced with some connected affine étale neighborhood $s \rightarrow U \rightarrow S_{0}=\operatorname{Spec}\left(R_{0}\right)$ of $s$. 
COROLlary 7.23. Let $\mathcal{O}$ be as in Theorem 7.20. Given any field $k$ and any complete discrete valuation ring $R$ with residue field $k$, any torus with endomorphisms by $\mathcal{O}$ over $k$ can be realized as the special fiber of some semiabelian scheme over some connected affine étale neighborhood $s \rightarrow U \rightarrow S_{0}$ of $s$ that is generically an abelian variety with endomorphisms by $\mathcal{O}$ (which can be principally polarized) that is absolutely simple except in the two cases (A) and (B) in Proposition 7.9.

By combining Corollaries 7.21 and 7.23, we obtain the following strengthening of our main theorem.

THEOREM 7.24. In Theorem 1.3, suppose $T$ has endomorphisms by $\mathcal{O}$, where $\mathcal{O}$ is as in the beginning of this $\S 7$, but with the additional assumption that $B=\mathcal{O} \otimes_{\mathbb{Z}} \mathbb{Q}$ is a division algebra. Then we may assume that the semi-abelian scheme $G$ also has endomorphisms by $\mathcal{O}$ and, instead of (2) in Theorem 1.3, that $G_{\eta}$ is absolutely simple except in the two cases $(A)$ and $(B)$ in Proposition 7.9.

Remark 7.25. Conversely, as mentioned in the introduction, by [19, IX, 1.4], [7, Ch. I, Proposition 2.7], or [14, Proposition 3.3.1.5], any endomorphism structure on the generic fiber $G_{\eta}$ necessarily and uniquely extends to the whole semi-abelian scheme $G$, and also (by reduction) to the torus $T$ we stared with. Hence, there has been no loss of generality in considering only tori with endomorphisms by the same orders.

Remark 7.26. Certainly, there are many examples of orders $\mathcal{O}$ (with positive involutions) defining endomorphism structures of abelian varieties of certain dimension $n$, but cannot possibly have actions on any $n$-dimensional tori. For example, already when $n=1$, no CM elliptic curve can degenerate to any onedimensional torus. In fact, one can generalize this to show that certain abelian varieties must have potential good reductions everywhere: see [11, §4.2] (and the review of the literature there) for a systematic discussion.

Remark 7.27. The two exceptional cases (A) and (B) in Proposition 7.9, and therefore also in Theorems 7.20, 7.22, and 7.24, correspond to the two exceptional cases (b) and (d) in [20, $\$ 4$, Theorem 5], which are treated in more detail in [20, §4, Propositions 17 and 18]. These results in [20, §4] are relevant for our investigation of degenerations of abelian varieties into tori, because the corresponding complex moduli can be non-compact, and because their toroidal compactifications can be compared with the corresponding ones in [14, Theorem 6.4.1.1] in a way that respects the degenerations of abelian varieties into semiabelian varieties along the boundary, by [12, Theorem 4.1.1 and Proposition 4.2.2]. (More precisely, some subcases of the two exceptional cases (b) and (d) in [20, $\$ 4$, Theorem 5] are still irrelevant, because the corresponding complex moduli are compact, and therefore cannot parameterize non-trivial degenerations of abelian varieties, by [11, §4.2]. Similarly, the remaining exceptional cases (a), (c), and (e) in [20, §4, Theorem 5], which are treated in more detail in [20, $\S 4$, Propositions 14, 15, and 19], are also irrelevant, because the corresponding complex moduli are compact.) 
Acknowledgements. We would like to thank the anonymous referees for their helpful comments.

\section{References}

1. M. Artin, Algebraic approximation of structures over complete local rings. Publ. Math. Inst. Hautes Études Sci. 36 (1969), 23-58.

2. C. Birkenhake and H. Lange, Complex Abelian Varieties, 2nd, augmented edn. (Grundlehren der Mathematischen Wissenschaften 302), Springer (Berlin, 2004).

3. S. Bosch, W. Lütkebohmert and M. Raybaud, Néron Models (Ergebnisse der Mathematik und ihrer Grenzgebiete, 3. Folge 21), Springer (Berlin, 1990).

4. C. W. Curtis and I. Reiner, Representation Theory of Finite Groups and Associative Algebras, AMS Chelsea Publishing, American Mathematical Society (Providence, RI, 1962); reprinted in 2006.

5. P. Deligne and M. Rapoport, Les schémas de modules de courbes elliptiques. In Modular Functions of One Variable II (Lecture Notes in Mathematics 349) (eds P. Deligne and W. Kuyk), Springer (Berlin, Heidelberg, New York, NY, 1973), 143-316.

6. M. Demazure and A. Grothendieck (eds), Schémas en groupes (SGA 3), II: Groupes de type multiplicatif, et structure des schémas en groupes généraux (Lecture Notes in Mathematics 152), Springer (Berlin, 1970).

7. G. Faltings and C.-L. Chai, Degeneration of Abelian Varieties (Ergebnisse der Mathematik und ihrer Grenzgebiete, 3. Folge 22), Springer (Berlin, 1990).

8. A. Grothendieck (ed.), Revêtements étales et groupe fondamental (SGA 1) (Lecture Notes in Mathematics 224), Springer (Berlin, 1971).

9. A. Grothendieck and J. Dieudonné, Eléments de géométrie algébrique (Publications mathématiques de l'I.H.E.S. 4, 8, 11, 17, 20, 24, 28, 32), Institut des Hautes Etudes Scientifiques (Paris, 1960, 1961, 1961, 1963, 1964, 1965, 1966, 1967).

10. N. Jacobson, The Theory of Rings (Mathematical Surveys and Monographs 2), American Mathematical Society (Providence, RI, 1948) (reprinted with corrections).

11. K.-W. Lan, Elevators for degenerations of PEL structures. Math. Res. Lett. 18(5) (2011), 889-907.

12. K.-W. Lan, Comparison between analytic and algebraic constructions of toroidal compactifications of PEL-type Shimura varieties. J. Reine Angew. Math. 664 (2012), 163-228.

13. K.-W. Lan, Toroidal compactifications of PEL-type Kuga families. Algebra Number Theory 6(5) (2012), 885-966.

14. K.-W. Lan, Arithmetic Compactification of PEL-type Shimura Varieties (London Mathematical Society Monographs 36), Princeton University Press (Princeton, NJ, 2013).

15. S. Lang, Algebra, 3rd edn., Addison-Wesley (Reading, MA, 1993).

16. W. Lütkebohmert, Rigid Geometry of Curves and their Jacobians (Ergebnisse der Mathematik und ihrer Grenzgebiete, 3. Folge 61), Springer (Berlin, 2016).

17. D. Mumford, Abelian Varieties (Tata Institute of Fundamental Research Studies in Mathematics 5), Oxford University Press (Oxford, 1970); with appendices by C. P. Ramanujam and Yuri Manin.

18. D. Mumford, An analytic construction of degenerating abelian varieties over complete rings. Compos. Math. 24(3) (1972), 239-272.

19. M. Raynaud, Faisceaux amples sur les schémas en groupes et les espaces homogènes (Lecture Notes in Mathematics 119), Springer (Berlin, 1970).

20. G. Shimura, On analytic families of polarized abelian varities and automorphic functions. Ann. Math. (2) 78(1) (1963), 149-192.

21. J. Tate, The arithmetic of elliptic curves. Invent. Math. 23 (1974), 179-206.

Kai-Wen Lan,

University of Minnesota,

Twin Cities,

MN 55455,

U.S.A.

E-mail: kwlan@math.umn.edu
Junecue Suh,

University of California,

Santa Cruz,

CA 95064,

U.S.A.

E-mail: jusuh@ucsc.edu 